Executive Function in Preschoolers with Primary Language Impairment by

\title{
Hui-Chun Yang
}

\author{
A Dissertation Presented in Partial Fulfillment \\ of the Requirements for the Degree \\ Doctor of Philosophy
}

Approved July 2015 by the Graduate Supervisory Committee:

\author{
Shelley Gray, Chair \\ Maria Restrepo \\ Tamiko Azuma \\ Samuel Green
}

\section{ARIZONA STATE UNIVERSITY}

August 2015 


\begin{abstract}
Research suggests that some children with primary language impairment (PLI) have difficulty with certain aspects of executive function; however, most studies examining executive function have been conducted using tasks that require children to use language to complete the task. As a result, it is unclear whether poor performance on executive function tasks was due to language impairment, to executive function deficits, or both. The purpose of this study is to evaluate whether preschoolers with PLI have deficits in executive function by comprehensively examining inhibition, updating, and mental set shifting using tasks that do and do not required language to complete the tasks.

Twenty-two four and five-year-old preschoolers with PLI and 30 age-matched preschoolers with typical development (TD) completed two sets of computerized executive function tasks that measured inhibition, updating, and mental set shifting. The first set of tasks were language based and the second were visually-based. This permitted us to test the hypothesis that poor performance on executive function tasks results from poor executive function rather than language impairment. A series of one-way analyses of covariance (ANCOVAs) were completed to test whether there was a significant between-group difference on each task after controlling for attention scale scores. In each analysis the between-group factor was group and the covariate was attention scale scores.

Results showed that preschoolers with PLI showed difficulties on a broad range of linguistic and visual executive function tasks even with scores on an attention measure covaried. Executive function deficits were found for linguistic inhibition, linguistic and visual updating, and linguistic and visual mental set shifting. Overall, findings add to evidence showing that the executive functioning deficits of children with PLI is not
\end{abstract}


limited to the language domain, but is more general in nature. Implications for early assessment and intervention will be discussed. 


\section{DEDICATION}

This dissertation is dedicated to my sweet and loving Father and Mother 


\section{ACKNOWLEDGMENTS}

I would like to express my gratitude to my committee members, Dr. Tamiko Azuma, Dr. Samuel Green, and Dr. Maria Restrepo for their constructive feedback on the initial proposal that led to the culmination of this final project. I would like to thank my research assistants, without whom, none of this research would have been possible. I would also like to thank all the school districts and families who were willing to participate in my study.

A special thanks goes to my doctoral friends in the trenches with me, Michael Tat, Ileana Ratiu, Nora Schlesinger, Elizabeth Runnion, Carol Mesa, and the co-workers from the Child Language and Literacy Lab. Thank you all for always being there for my ups and downs throughout this tough journey. I am deeply grateful to have had all of your companionship and support for these five years. You are my family here.

I would like to thank my family, teachers and friends from Taiwan. They were always cheering me up, encouraging me with their best wishes and stood by me through the good times and bad.

Finally, I would like to give my deepest thanks to my supervisor, Dr. Shelley Gray, whose expertise, understanding, generous and patient guidance made it possible for me to work on a topic that is of great interest to me. A simple thank you does not enough for all the support and mentoring throughout $\mathrm{PhD}$ program. You have helped me become a competent researcher and a stronger person. I would not have made it this far without your guidance. You have taught me far more than I ever expected and for that I will always be grateful. 
LIST OF TABLES........................................................................................... vii

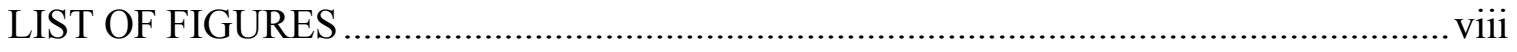

\section{CHAPTER}

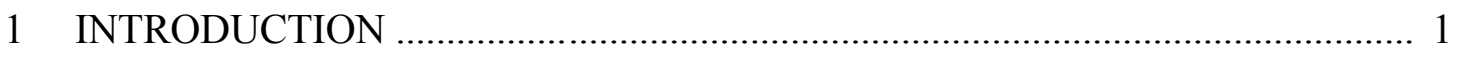

Executive Function in Preschoolers ................................................... 3

Three Theoretical Frameworks on Executive Function in Preschoolers . 6

The Organization of Executive Functions in Preschoolers .................... 8

Development of Prefrontal Cortex During the Preschool Period .......... 11

The Impact of Language on Executive Function in Preschool ............. 13

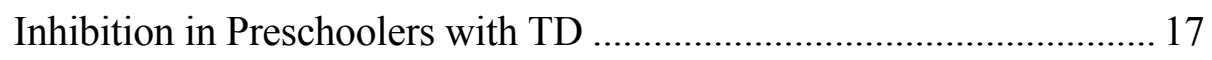

Inhibition in Preschoolers with PLI............................................... 20

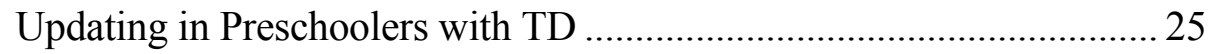

Updating in Preschoolers with PLI.................................................... 27

Mental set shifting in Preschoolers with TD ....................................... 27

Mental set shifting in Preschoolers with PLI ...................................... 31

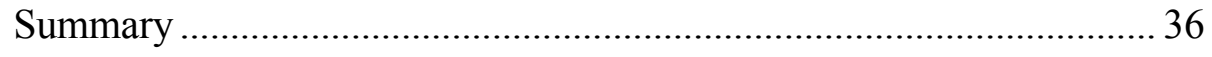

Purpose, Research Questions, and Hypotheses ................................. 38

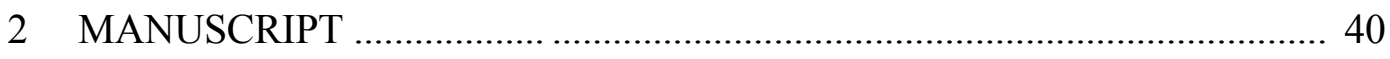

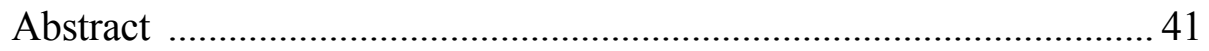

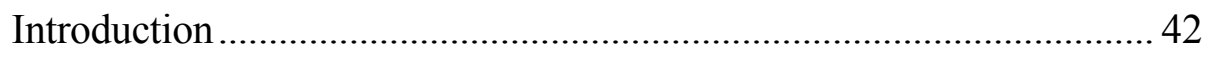

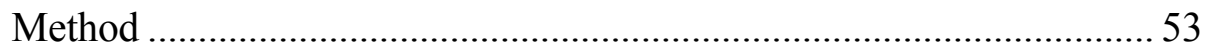


CHAPTER

Page

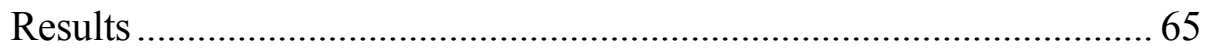

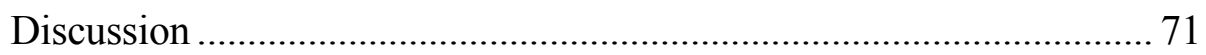

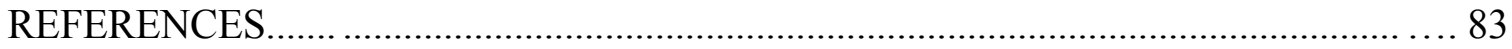

APPENDIX

A ARIZONA STATE UNIVERSITY'S INSTITUTIONAL REVIEW BOARD.... 93 


\section{LIST OF TABLES}

Table

Page

1. Comparison of Results between Linguistic and Nonlinguistic Executive Functions

Tasks in Preschoolers and School-Age Children with Primary Language

Impairment Relative to Age-Matched Peers with Typical Development.... 95

2. Participant Description Information Including Summary of Inclusionary Test Results

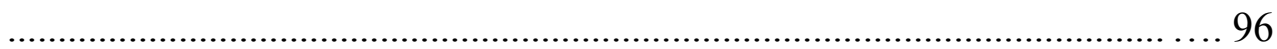

3. Descirption of Executive Function Tasks in Current Study ................................. 97

4. Reliability of Executive Function Tasks in Current Study .................................. 98

5. ANOVA Results of Groups on Experimental Tasks .......................................... 99

6. ANCOVA Results of Groups on Experimental Tasks ….................................... 100 


\section{LIST OF FIGURES}

Figure

Page

1. Ilustration of Executive Function Tasks Used in Current Study .............. 101 


\section{CHAPTER 1}

\section{INTRODUCTION}

Children with primary language impairment (PLI) are identified by their poor performance on language development measures despite having no hearing impairment, neurological damage, or intellectual disability (Leonard, 1998). Even though their main deficits are in the area of language development and acquisition, research also suggests that school-age children with PLI demonstrate difficulties in other cognitive processes (Ellis Weismer, Evans, \& Hesketh, 1999; Ellis Weismer, Plante, Jones, \& Tomblin, 2005; Hoffman \& Gillam, 2004; Marton, 2008; Spaulding, Plante, \& Vance, 2008; Windsor \& Kohnert, 2009), including aspects of executive function (Bishop \& Norbury, 2005; Dibbets, Bakker, \& Jolles, 2006; Henry, Messer, \& Nash, 2012; Im-Bolter, Johnson, Pascual-Leone, 2006; Marton, Kelmenson, \& Pinkhasova, 2007; Marton, 2008; Noterdaeme, Amorosa, Mildenberger, Sitter, \& Minow, 2001; Spaulding, 2010; Wittke, Spaulding, \& Schechtman, 2013).

Executive function, also called cognitive control, refers to the allocation of attentional resources involving high-level goal-directed behavior (Lyon \& Krasnegor, 1996). In general, it is considered a collection of interrelated abilities that enable individuals to modify their thoughts and actions (Baddeley, 1986; Norman \& Shallice, 1986). According to Miyake et al. (2000), there are three fundamental executive functions: inhibiting irrelevant responses, continuously updating information stored in working memory, and shifting between mental sets. Executive functions are associated with prefrontal cortex activity, which develops during the preschool years. It is important to study executive function during the preschool years because executive function is a 
strong predictor of vocabulary (McClelland et al., 2007) and school readiness skills such as early literacy (McClelland et al., 2007; Welsh, Nix, Blair, Bierman, \& Nelson, 2010), numeracy skills (Blair \& Razza, 2007; McClelland et al., 2007) and classroom-related behaviors and engagement (Brock, Rimm-Kaufman, Nathanson, \& Gimm, 2009) in preschoolers and kindergarteners.

Evidence regarding executive function in preschool-age children with PLI is limited. Most studies investigating executive function in these children have been restricted to the school-age population. In addition, most of these studies have been conducted using "linguistically-based" executive function tasks, which require children to use their linguistic knowledge to complete them. As a result, we do not know whether poor performance is due to language impairment, to executive function deficits, or both. A few studies have used "visually-based" tasks that did not require children to use linguistic knowledge to complete tasks, but the results were inconclusive. That is, children with PLI performed significantly poorer than their peers with typical development (TD) on some visually-based tasks (Bishop \& Norbury, 2005; Henry et al., 2012; Im-Bolter et al., 2006), but they performed comparable to their peers with TD on other visually-based tasks (Noterdaeme et al., 2001; Henry et al., 2012; Im-Bolter et al., 2006). To gain a better understanding of the nature of the executive function in children with PLI, a comprehensive study that tests performance on both linguistic and visual executive function tasks is needed. 


\section{Executive Function in Preschoolers}

"Executive function" is an umbrella term encompassing a set of general-purpose control mechanisms that regulate the dynamics of cognition and action (Lyon \& Krasnegor, 1996). Executive functions are the key components of information processing that enable the resolution of conflict and the maintenance of goal-directed behaviors. There is a debate about the nature of executive functions. Some researchers view executive functions as a unitary construct (Baddeley, 2012; Kane \& Engle, 2002), but most argue that executive functions are dissociable and can be fractionated into different functions, such as strategic planning, flexibility of thought and action, inhibition of inappropriate responses, generation of new responses, and concurrent remembering and processing. (Lehto, Juujarvi, Kooistra, \& Pulkkinen, 2003; Miyake et al., 2000).

The most widely known framework of executive function was proposed by Miyake et al., (2000). They viewed executive function as a general control mechanism that modulates cognition, with a focus on three executive functions: (a) inhibition of prepotent responses, (b) updating and monitoring of working memory representations, and (c) shifting between mental sets. Inhibition is the deliberate, controlled suppression of prepotent responses. Updating requires individuals to dynamically monitor and code incoming information based on relevance to the task and then revise the content held in working memory by replacing no longer relevant information with updated relevant information. Shifting between mental sets (task switching) involves the ability to engage and disengage from tasks and also the ability to perform a new operation in the face of proactive interference or negative priming. Miyake et al. asked 137 undergraduate students to complete nine tasks designed to tap one of the three target executive functions 
of inhibition, updating, and shifting, and five complex tasks commonly used as measures of executive function. Confirmatory factor analyses showed that inhibition, updating, and shifting were moderately correlated with one another, but they were also separable. Thus, the nature of these three executive functions includes both unity and diversity. This threecomponent framework has been applied to a large twin sample (Friedman, Miyake, Robinson, \& Hewitt, 2011), school-age children (Huizinga, Dolan, \& Molen, 2006; Lehto et al., 2003), and adults (Vaughan \& Giovanello, 2010).

To explain the correlations between inhibition, updating and mental set shifting, Miyake et al., (2000) proposed that the three target executive functions all involve some sort of inhibitory processes. For example, updating may require ignoring irrelevant incoming information and suppressing no longer relevant information. Shifting may require suppressing an old mental set to switch to the new set. This proposal was supported by Friedman et al. (2011) when they tracked a twin sample's performance on self-restraint indexed by prohibition tasks where children were told not to touch an attractive toy for 30 seconds. Children ranged in age from 14 months to 3 year of age and were assessed at four time points $(14,20,24$, and 36 months of age). Response latency was the dependent variable. Additionally, when they were 17 years old, their inhibition, updating, and mental set shifting were measured using the same set of executive function tasks used in Miyake et al. The first key finding was that based on confirmatory factor analysis, performance on the three executive functions could be decomposed into (a) a common executive function, which contains an inhibition component, (b) an updatingspecific component, and (c) a shifting-specific component. The second key finding was that growth modeling of the latency at four time points showed two distinct 
developmental trajectories (children with better vs. worse self-restraint, and this betweengroup difference remained at 17 years. The third finding was that children with better self-restraint demonstrated significantly better common executive function, no difference in the updating-specific ability, and significantly worse shifting-specific ability than did children with worse self-restraint. These results not only highlighted the unity/diversity nature of these three executive functions, but also revealed the fundamental role of inhibition, which supports the proposal of Miyake et al. Furthermore, it is noteworthy that individual differences in self-restraint and executive function were relatively stable in development, which may imply that if young children have poor executive function early in their development, there is a high possibility that they may continue demonstrating poor executive function later. Thus, the early developmental stage of executive function is important and establishes the foundation for the later development.

The first five years of life play a critical role in the development of executive function (Garon, Bryson, \& Smith, 2008). The core executive functions of inhibition, updating and mental set shifting develop during this period and establish a fundamental base for the development of other higher cognitive processes in adulthood (Best \& Miller, 2010; Garon et al., 2008). However, compared to our understanding of executive function in adults and school-age children, we have less knowledge about the development of executive function in preschoolers. The following section will first review contemporary theoretical frameworks of executive function in preschoolers and how these frameworks account for developmental change in executive function. The review will then focus on neurological evidence related to executive function development in preschoolers, 
followed by a review of inhibition, updating, and mental set shifting in preschoolers with TD and PLI.

\section{Three Theoretical Frameworks on Executive Function in Preschoolers}

There are three major frameworks specifically addressing executive function in preschoolers. The first is the Cognitive Complexity and Control theory proposed by Zelazo and Frye (1998), which views executive function as a functional construct of the psychological processes involved in goal-directed problem solving. Rather than defining different functions involved in executive functioning, they define executive functions in terms of what they accomplish. They emphasize the importance of complexity.

Complexity is defined in terms of the hierarchical structure of children's rule systems.

The structure of this hierarchy can be formed by one rule being embedded within another higher order rule and controlled by it. For example, children learn a rule that links antecedent conditions to consequences (i.e., if I see a mail box, then I need to mail this letter). When children acquire the ability to reflect on the rules they represent, they become able to consider them in contrast to other rules (i.e., if I don't see a mail box, then I will go to the post office to mail this letter) and embed them under higher order rules (i.e., if it is before 5 p.m.). The development of executive function is shown by increases in the maximum complexity of the rules children can formulate and use when solving problems (Zelazo, Müller, Frye \& Marcovitch, 2003). Thus, as children's rule systems increases in complexity during the preschool years, they are more able to control their reasoning and behaviors. According to this account, if a child has difficulties with problem solving this might be explained as a kind of representational inflexibility 
because the child's rule structure is not complex enough to reflect the situation. He can only apply the limited rules he has.

The second theoretical framework is Graded Representation, first proposed by Munakata (2001), which views executive functions as the ability to act flexibly in changing circumstances and to avoid prepotent behaviors that are no longer appropriate. Morton and Munakata (2002) proposed a later model composed of two distinct representations, active versus latent. Latent representations develop earlier and reflect gradual learning. They are formed in the posterior cortex when processing a stimulus brings about a change in the subsequent processing of the stimulus, which is more associated with long-term memory. Active representations develop later and provide topdown control. They are formed in the prefrontal cortex when organisms actively maintain a representation of a specific stimulus and manipulate information, which is more associated with working memory and attention. Accordingly, young children often behave inflexibly in changing circumstances by repeating inappropriate prepotent behaviors because their active representation for currently relevant information is not strong enough to compete against their latent representation for previously relevant information. Developmental change is reflected by increasingly strong representations in active memory (Munakata, 2001). Children become better able to act appropriately in response to changing contextual demands rather than perseverating on prepotent responses. Thus, if a child has difficulty applying new rules to a task, his ability to actively maintain current rules is insufficient to overcome the previous rule, which makes him act inflexibly in the changing context. 
The third theoretical framework is the Multifactoral Framework, proposed by Diamond (2006), which defines executive function as the ability to use a representation to guide behavior despite the pull of previous experience. Executive function is utilized when conscious, cognitive control is required, as opposed to occasions when prepotent behaviors would be sufficient. She proposed three executive function components: (a) inhibition, the ability to ignore distraction and to resist making one response and instead make another; (b) working memory, the ability to hold information in mind and manipulate it; and (c) cognitive flexibility (also known as shifting), the ability to flexibly switch perspectives or response mappings. Diamond (2006) suggested that these three executive functions show different developmental trajectories and they interact with one another. She noted that between 3 and 5 years there are remarkable improvements in inhibition and cognitive flexibility. Diamond (2006) also suggested that inhibition control is a central feature of the development of executive function in early childhood.

\section{The Organization of Executive Functions in Preschoolers}

Many developmental studies have adopted the multifactorial framework of Miyake et al., (2000), which differentiated inhibition, updating and mental set shifting. The majority studies that focus on organization in preschoolers found a single factor framework and failed to find the three-component framework (Shing, Lindenberger, Diamond, Li, \& Davidson, 2010; Wiebe, Espy, \& Charak, 2008; Wiebe et al, 2011; Willoughby, Blair, Wirth, \& Greenberg, 2012). These findings suggested that executive function is unitary in preschoolers and that executive function is not fractionated until young adulthood, around 15 years of age (Lee, Bull, \& Ho, 2013). For example, Wiebe, Espy and Charak (2008) used confirmatory factor analysis to examine executive function 
in 243 preschoolers between 2.25 and 6 years of age by asking them to complete a battery of executive function tasks that tapped working memory and inhibition. Their best fitting model had a single-factor and this same unitary model fit subgroups of children divided by socioeconomic status and by gender. Further confirmatory factor analyses with data from a sample of 3-year-olds, who completed several measures of working memory and inhibition, also confirmed the validity of this unitary executive function model (Wiebe et al., 2011).

However, Miller, Giesbrecht, Muller, Mclnerney, and Kerns (2012) argued that task selection and performance indications (dependent variables) may have influenced the findings on organization of executive function in preschoolers. They argued that Wiebe and colleagues $(2008,2011)$ may not have found an inhibition factor for preschoolers because their selected inhibition tasks did not involve a strong prepotent response. For example, Wiebe et al., (2008) used a delayed responses task to measure inhibition, but it involved only a 10-second delay. Wiebe et al., (2011) used accuracy as a performance indicator for their Go/Nogo task, but the accuracy scores were combined for both 'go' and 'no-go' trials and they did not include a mental set shifting task. Miller et al., (2012) asked 129 children between 3 to 5 years of age to complete a battery of executive function tasks that included mental set shifting and performance indicators that more clearly separated working memory and inhibition demands. Their confirmatory factor analyses showed a two-factor model of executive function with better fit and model comparison values than a single-factor model. This two-factor model included two significantly correlated components, a component of inhibition and a component that combined updating and mental set shifting. Their explanation of why mental set shifting 
loaded on the working memory factor was that the mental set shifting indicators contained properties of working memory because children had to actively maintain a current rule in mind to successfully shift from a previous rule. Results of this study revealed that working memory (updating) and inhibition are separable components of executive function in preschoolers, which supported Diamond's (2002) proposal that working memory and inhibition are dissociated in young children. Additionally, the high correlation between the component of inhibition and the component that combined updating and mental set shifting suggested that young children's executive function is similar to the unity and diversity of adults' executive function (Miyake et al., 2000).

The multifactorial framework of executive function in preschoolers has also been supported by Hughes (1998). Hughes asked 50 preschoolers with a mean age of 3 years 11 months to complete six executive function tasks that tapped working memory, inhibitory control, and attentional flexibility (e.g., shifting). The author used principal component analysis to analyze whether distinct aspects of executive function could be differentiated. The analysis extracted these three executive functions, which together explained 79 percent of the remaining variance in the data set. Results support Diamond's (2006) view of the distinction between inhibition, working memory, and attentional flexibility.

Taken together mixed research findings suggest either a single-factor framework of executive function in preschoolers or dissociable functions including inhibition, updating, and mental set shifting. Thus, a multifactorial organization of executive function should not be ruled out. It is necessary to investigate individual differences in each executive function because different developmental disorders may involve deficits 
in different aspects of executive function. Examining each identified executive function allows us to determine whether there are different profiles of executive functions in preschoolers with PLI and TD.

\section{Development of Prefrontal Cortex during the Preschool Period}

Neurologically, Luria (1973) proposed that executive functions are related specifically to the prefrontal cortex based on her observations of patients with prefrontal cortex damage. Cognitive neuroscience evidence suggests that the prefrontal cortex contributes to retrieval control, higher cognitive functions (e.g. planning, reasoning, language, and general intelligence), and that it plays an important role in integrating information from many areas of the brain. Findings from adult neuroimaging studies support the multicomponent nature of executive function (Collette \& der Linden, 2002; Rypma \& D'Esposito, 2000). Different components of executive function are associated with different parts of the prefrontal cortex. For example, the ability to inhibit responses has been found to rely on the orbitofrontal cortex (Roberts \& Wallis, 2000). The ability to maintain and manipulate information in working memory is thought to recruit primarily lateral prefrontal cortex (Rypma \& D’Esposito, 2000), and the ability to switch between tasks has been found to rely on the medial prefrontal cortex (Crone, Wendelken, Donohue, \& Bunge, 2005). Therefore, different executive functions appear to recruit different regions within the prefrontal cortex.

Accumulating and converging evidence indicates that early childhood is important for functional neural development of the prefrontal cortex (Espy, 2004) and that the prefrontal network is not fully established until young adulthood (Tsujimoto, 2008). Prefrontal cortex maturation during early childhood is characterized by a reduction 
of synaptic and neuronal density, the growth of dendrites, and increased volume in gray and white matter (Anderson, Jacobs, \& Anderson, 2008; Tsujimoto, 2008).

Two neuroimaging studies in young children have addressed prefrontal cortex function in young children. To investigate developmental changes in the prefrontal cortex, Tsujimoto, Yamamoto, Kawaguchi, Koizumi, and Swaguchi (2003) used nearinfrared spectroscopy, which is a critical index of neural activation, to study prefrontal activities in children with a wider age range ( 4 years, 4 months to 6 years, 8 months). Children were asked to complete a working memory task that required them to remember the locations of a sample cue array during a delay period of eight seconds then correctly report whether the location of a test cue was identical to any of the locations of the sample cue. Results showed that the activation level increased gradually as a function of age from 4 to 7 years and age (in months) and was positively correlated with the activation level. They also conducted a multiple linear regression analysis that included age, accuracy, and reaction time as predictors of activation level. Results showed that age was the most significant predictor of prefrontal cortex activity. This study provided direct evidence for the development of working memory and its corresponding increased activity in the lateral prefrontal cortex in children between four and seven years of age. Tsujimoto, Yamamoto, Kawaguchi, Koizumi, and Swaguchi (2004) also used the same technique to study prefrontal activities in normal adults and children ages five and six years with TD. Participants were asked to complete a same working memory task. Results showed that children activated the same region of the prefrontal cortex, the lateral prefrontal cortex, as adults during this task. 
The two studies above provided two significant findings: (a) that the lateral prefrontal cortex, which is responsible for working memory, already functions in children as young as four years with TD; and (b) it gradually matures from four to seven years of age. Results showed that working memory already mapped onto a specific region of prefrontal cortex in four-year-olds; thus, it is possible that inhibition and mental set shifting may also begin to map onto specific parts of the prefrontal cortex in young children. Therefore, it is important to gain more information about each executive function during this crucial period because each function in preschoolers matures from infancy to childhood (Garon et al., 2008; Tsujimoto, 2008).

\section{The Impact of Language on Executive Function in Preschool}

Increasing evidence points to a positive relationship between executive function and language development in preschoolers. For example, in a study of 50 preschool children Hughes (1998) found significant correlations between verbal ability, including receptive and expressive language abilities and inhibitory control, and between verbal ability and working memory after age was partialled out. Carlson, Mandell, and Williams (2004) found a significant correlation between performance on behavioral batteries of executive function and parent reports of language skills in 2- and 3-year-olds. Moreover, Wittke et al. (2013) also identified a significant correlation between scores on the Behavior Rating Inventory of Executive Function-Preschool (Gioia, Espy, \& Isquith, 2003), parent and teacher ratings, and language abilities as assessed by the Clinical Evaluation of Language Fundamentals Preschool-Second Edition (CELF P-2; Wiig, Secord, \& Semel, 2004) in preschoolers with TD and with PLI. 
A number of researchers favor the view that language development enables children to organize and plan their behavior. Vygotsky (1987) hypothesized that parents initially regulate young children's behaviors through verbalizations, and children begin to self-regulate by overt verbalizations of their own and later by inner speech (internalized verbal thought). This self-regulatory function of language (a.k.a., private speech) emerges between the ages of three and five years, which potentially fosters the development of executive function during this period (Zelazo et al., 2003). Accordingly, language serves an instrumental role in consciousness and behavior control (Zelazo, 1999). Preschoolers learn to use language for self-instruction and to scaffold their understanding (Homer, Petroff, \& Hayward, 2008).

Alarcon-Rubio, Sanchez-Medina, and Prieto-Garcia (2013) support the above account. They investigated the relationship between private speech and mental set shifting in children between four- and seven-year-olds. Mental set shifting was measured by the Dimensional Change Card Sorting Task (DCCS; Frye, Zelazo, \& Palfai, 1995) that required children to switch between card sorting rules. Private speech was observed during the categorization task, which asked children to verbally sort 25 cards into five categories. They found a developmental trend in both private speech and the shifting task between ages four and seven years. Logistic regression models showed that age (in months) and the verbal ability score, as indexed by the Peabody Picture Vocabulary TestRevised (PPVT-R; Dunn \& Dunn, 1997), were the main factors associated with the children's performance on the shifting task. Results from linear regression models showed that age and verbal ability accounted for the greatest amount of variance in the number of correct items on the DCCS task. After controlling for age, gender, verbal 
abilities, and fluid intelligence, the rate of private speech per minute was significantly associated with the number of blocks that children shifted rules on successfully. These results confirmed that children as young as age four were able to formulate language rules based on their knowledge and one task demands (e.g., "In color games, I will put the red card to here and put the blue card to there"). Further, children who used more frequent private speech were more likely to correctly switch between the sorting rules. This study provides evidence that young children can use language as a cognitive instrument for guiding, planning, and regulating their own thoughts and behavior.

Other researchers have focused on the impact of verbal labeling on the DCCS task (Kirkham, Cruess, \& Diamond, 2003; Müller, Zelazo, Lurye, \& Liebermann, 2008). Kirkham, Cruess, and Diamond (2003) compared the performance of three-year-olds on a standard version of DCCS task and a labeled version. In the labeled condition they asked children to label the test card's current sorting dimension on the first trials of each dimension. For example, before the experimenter asked "Where does this card go?" the experimenter asked the child, "What's this one?" and waited for the child to answer "a truck" in the shape dimension or "blue" in the color dimension. They found that the performance of shifting to the other dimension was significantly better in the labeled version than the standard version in three-year-olds. However, there is no clear reason why verbal labeling assisted three-year-olds on this task. It may that labeling facilitates the ability to reflect on the rule of sorting (Happaney \& Zelazo, 2003), that labeling increases the amount of time that the rule remains in working memory (Munakata, Morton, \& Yerys, 2003), or perhaps labeling helps direct the focus of attention by 
scaffolding children's ability to inhibit their focus on the previous rule (Kirkham et al., 2003).

The impact of language on executive function development in preschoolers has also been confirmed in a longitudinal study. Fuhs and Day (2011) examined whether verbal ability is a promising predictor of executive function development in 132 children between 43 and 63 months of age during the fall, then followed up in the spring. Children were asked to complete two inhibition and two shifting tasks. Children's verbal ability was measured using the Picture Naming, Receptive Vocabulary, and Information subscales from the Wechsler Preschool and Primary Scale of Intelligence (Wechsler, 2002). Both executive function and language measurements were assessed in the fall and spring. The authors used structural equation modelling to examine the longitudinal relationship between verbal ability (composite score of three language measurements) and executive functions (composite score of inhibition and shifting tasks). The model revealed that verbal ability in fall was a significant predictor of executive function in spring after controlling for fall executive function and age. Fall executive function was a significant predictor of spring executive function, and fall verbal ability was a significant predictor of spring verbal ability. Furthermore, they tested a potential bidirectional relationship between verbal ability and executive function development. Result showed that executive function in fall was not a significant predictor of verbal ability in spring. This study showed that verbal ability, assessed in the fall, predicts individual variations in longitudinal development of inhibition and shifting in preschoolers.

Combined, these studies suggest that language ability plays a role in the development of executive function in preschoolers. Language may provide the function 
of self-regulation that supports preschooler's ability to regulate their thoughts and behaviors. It may also allow preschoolers to use verbal labeling to facilitate processing of current relevant information and to maintain it in working memory. Following this logic, it may reasonable to assume that children would perform better on executive function tasks that use linguistic stimuli than executive function tasks that used nonlinguistic stimuli because linguistic stimuli are easier to label and regulate using language than tasks using nonlinguistic stimuli. Furthermore, if preschoolers have poor language ability, their language impairment may negatively impact their executive functions. They may find it difficult to use the labeling and self-regulatory functions of language when they complete executive function tasks. Because preschoolers with PLI may not benefit from language as much as children with TD, the performance difference between preschoolers with PLI and TD may be larger on executive function tasks that use linguistic stimuli than on tasks that use non-linguistic stimuli.

The following literature review of executive functioning in preschoolers is based on the widely used framework proposed by Miyake et al., (2000). This framework includes inhibition, updating and mental-set shifting. The literatures regarding executive function in preschoolers with TD will be reviewed first, followed by literature on both linguistically-based and non-linguistically-based executive function tasks in preschoolers with PLI (see Table 1 for the summary of all the studies regarding executive functions in children with PLI).

\section{Inhibition in Preschoolers with TD}

Critical developments in inhibition take place between ages three and six (Garon et al., 2008). Children gradually become more able to suppress automatic responses 
during the preschool years in terms of the length of time that they can suppress their automatic responses. This ability is usually assessed using simple response inhibition tasks. For example, in the delay of gratification paradigm, children are asked to choose whether they want to get a reward now or wait longer for a bigger reward. Carlson (2005) found age differences in a cross-sectional sample of two- to four-year-olds in the length of time that children were able to delay reward. Eighty-five percent of three-year-olds suppressed the desire for one minute, whereas $72 \%$ of four-year-olds suppressed for five minutes. These results revealed that the ability to suppress lengthens throughout the preschool period.

The ability to inhibit a dominant response, which helps hold an arbitrary rule in mind and respond according to the rule, also improves significantly during the preschool years. This ability was observed on preschoolers' performance in Stroop-like tasks that involved inhibition of a strong prepotent response. Guy, Rogers and Cornish (2012) used a Cat-Dog task to examine age-related changes in visual and auditory inhibition in 68 preschoolers with TD ages three to six years. In the visual inhibition task children were asked to say "cat" when they saw a picture of a "dog" on the screen and say "dog" when they saw a picture of a "cat." In the auditory inhibition task children were asked to say "cat" when they heard a dog barking and to say "dog" when they heard a cat meowing. They found a similar developmental improvement in both auditory and visual inhibition tasks in terms of accuracy. The three-year-olds were the least accurate and slowest to respond with more errors, non-responses, and more incorrect responses than older-age children. They also found a rapid increase in inhibition in terms of accuracy and response 
time (RT) between the ages of three to five years, but only modest improvement thereafter.

Additional evidence suggests that the ability to inhibit distractor interference and prepotent responses does not develop completely during preschool. Rueda, Posner, Rothbart and Davis-Stober (2004) used a Flanker paradigm to examine developmental differences in conflict processing among 22 four-year-old children with TD and 18 adults. The Flanker paradigm requires participants to respond only to the target stimuli and inhibit distraction from flankers. The flankers are either congruent, indicating the same response as the target, or incongruent, indicating the different response from the target. The idea is that both target and flankers are processed. The congruent flankers prime a correct response and the incongruent flankers prime an incorrect response. In other words the incongruent flankers generate interference (i.e., conflict) compared to the congruent flankers, which requires a longer RT because of inhibition and this may result in reduced accuracy. Rueda et al.'s results showed that children needed significantly longer to inhibit conflict interference than adults and children were less accurate than adults, which indicated that four-year-olds have greater difficulties in inhibiting conflict interference than adults.

Davidson, Amso, Anderson, and Diamond (2006) used a Simon task paradigm to document developmental changes in inhibition from four to 13 years of age and in young adults. The Simon task paradigm assesses whether non-spatial information, such as direction or color, is relevant to a task and whether its spatial location is irrelevant and needs to be inhibited. The Simon effect is that responses are faster and more correct when the stimulus and responses are on the same side (congruent trials) than when they are on 
opposite sides (incongruent trials). The logic is that participants have to inhibit both the influence of an irrelevant aspect of the stimulus and a prepotent intention of responding on the same side as the stimulus in incongruent trials. Davidson et al. found that the Simon effect was present at all ages and especially obvious in the younger group (four to six years of age). The youngest group showed the largest Simon effect among all the groups. Children four to five years showed $80 \%$ accuracy on incongruent trials, whereas, the older groups were able to perform above $85 \%$ accuracy on incongruent trials. Results indicated that four- and five-year-olds have more difficulty inhibiting irrelevant aspects of a stimulus and a prepotent intention to respond on the same side as the stimulus in incongruent trials than older children. Their results revealed not only that inhibition ability develops significantly between age four to six, but also that preschoolers have a harder time inhibiting irrelevant and prepotent tendencies than older children and adults.

\section{Inhibition in Preschoolers and School-Age Children with PLI.}

Only one study has shown that preschoolers with PLI have difficulties on inhibition tasks that involve linguistic processing. Spaulding (2010) investigated two inhibition mechanisms, intentional inhibition of prepotent responses and resistance to distracter interference, in preschoolers with and without PLI. Inhibition of prepotent responses was measured using a Stop-Signal paradigm that required children to press a button with a picture of a dinosaur when they heard the word "dinosaur" and to press a button with a picture of a butterfly when they heard the word "butterfly." The children were asked to hold their response when they heard the word "stop" following the presentation of the word. The resistance-to-distracter interference task asked children to press a button when they heard the target "give me ice cream," but to not respond to the 
non-target "give me pizza." Children were also asked to inhibit three types of distracters that were randomly presented prior to the target. The types of distractors were linguistic (i.e., sentences spoken with the same stress location and general affective tone), nonverbal auditory (i.e., environmental stimuli), and visual distracters (i.e., pictures). They found that preschoolers with PLI performed significantly less accurately than their TD peers on the Stop-Signal task. This supports the idea that preschoolers with PLI have more difficulty inhibiting prepotent responses than their peers with TD. Results for the resistance-to-distracter interference task revealed that preschoolers with PLI made significantly more errors and responded significantly slower than their peers with TD on all three types of distracter trials. There were no within-group differences in accuracy among the types of distracters. Both groups responded slowest on linguistic distractor trials. These results indicated that preschoolers with PLI had a hard time inhibiting irrelevant information, regardless of whether it was visual, nonverbal auditory, or linguistic in nature. Spaulding concluded that preschoolers with PLI have inefficient inhibition. She suggested that this may have a negative impact on the use of attentional resources because children may be unable to prevent the encoding of irrelevant information and/or discard previously encoded, but no longer relevant, information (Bjorklund \& Harnishfeger, 1990). These results suggest that preschool-age children with PLI exhibit inefficient inhibition.

An inhibition deficit in preschoolers with PLI has not been confirmed by all studies using inhibition tasks that involve linguistic processing. Henry et al. (2012) examined linguistic inhibition in school-age children with PLI (mean age 11 years). Their linguistic inhibition task was a Stroop-like task that required children to say "car" when 
the researcher said "doll" and vice versa. Although children with PLI performed more poorly than their TD peers, the between-group difference was not significant.

Most of the findings for school-age children with PLI also indicate that they demonstrate poor performance on inhibition tasks that involves linguistic processing. Marton et al. (2007) used a Listening Span task to measure inhibition ability in children with PLI (mean age 8;9), age-matched children with TD (AM), and language-matched children with TD (LM). This task required children to process linguistic information by asking them to comprehend simple sentences (i.e., answer yes or no question about each sentence), and to store verbal information by asking them to remember the last word of each sentence. The authors categorized children's errors into four types of inhibition errors: immediate (from the current set), delayed (from a previous set), contextual distraction (non-target items from the middle of the sentence), and perseveration (repeated a previously recalled word). Their results showed that children with PLI performed poorly in listening span tasks and produced more inhibition errors than their $\mathrm{AM}$ and LM peers. Furthermore, the within-group comparisons indicated that all children produced more immediate than delayed errors, but children with PLI made more immediate inhibition errors than both groups of children with TD. This means that children with PLI had difficulty suppressing words that were previously relevant, but were not relevant anymore. The authors also evaluated whether inhibition errors reflected contextual distraction, which were non-target items from the sentences, or perseveration, which were the previously recalled words. All participants produced more contextual distraction errors than perseverations, but children with PLI produced more errors in both 
categories than the children with TD. Overall, children with PLI demonstrated difficulties inhibiting irrelevant linguistic information.

Some studies have investigated inhibition in school-age children by using tasks that do not require linguistic processing. Children with PLI exhibited inhibition deficits in most of the experiments. For example, Bishop and Norbury (2005) used two tasks to measure children's inhibition. The first one entitled "Opposite Words" assessed response inhibition in children with and without PLI (age range six to10 years). This task required children to say "two" when seeing the number "one," and "one" when seeing the number "two." The second task was a Stop-signal paradigm, the "Walk, Don't Walk" task, which measured sustained attention that required inhibition of a non-verbal response. Children had to mark on footprints when they heard a target tone, but hold their response when they heard a warning signal. These two measures both involved inhibition of a prepotent response. In both tasks children with PLI made more errors than children with TD. This indicated that children with PLI had difficulty inhibiting the propotent verbal response, and they also had difficulty inhibiting a proponent response, even when no overt verbal response was required from them.

Henry et al. (2012) examined nonlinguistic inhibition in school-age children with PLI using a nonverbal motor task. This task required children to make hand gestures. For example, if the examiner pointed a finger, children needed to make a fist, and vice versa. Children with PLI made significantly more errors than children with TD on this nonlinguistic task. Similar findings have also been reported on an antisaccade task, which was designed to inhibit reflexive or automatic responses. Im-Bolter et al. (2006) used an antisaccade task to assess inhibition in school-age children with TD and with PLI. For 
each trial a visual cue was presented on one side of the screen, followed by a target stimulus, an arrow, appearing on the opposite side of the screen. Because the target stimulus appeared on the screen for a very short period of time, children had to inhibit the dominant responses of looking at the initial visual cue to indicate the direction of the arrow. Results showed that children with PLI made significantly more errors than children with TD, but there were no significant between-group differences in RT. Together, the results of these inhibition studies suggest that children with PLI have weaknesses in inhibition, even when the inhibition tasks do not require linguistic processing.

Although it appears that school-age children with PLI have difficulty with nonlinguistic inhibition tasks, this is not always the case. Noterdaeme, Amorosa, Mildenberger, Sitter, and Minow (2001) used two nonlinguistic inhibition tasks, a Go/No Go task and an Incompatibility task, to assess inhibition ability in school-age children with and without PLI. In the Go/No Go task, which was designed to test children's ability to inhibit responses to irrelevant stimuli, children were required to press the key when a " $\times$ " was presented on the screen and to refrain from pressing the key when a "+" was presented. An incompatibility task, also known as the Simon task, was designed to assess inhibition of prepotent responses. An arrow (pointing left or right) was either presented left or right of the central fixation point on the screen. Children were asked to press the right key when the arrow pointed to right and to press the left key when the arrow pointed to the left, regardless of where the arrow was presented. Thus, inhibition was required when the locations of stimulus and response were incompatible. Results showed no between-group differences in either accuracy or RT. These results are inconsistent with 
previous findings by Bishop and Norbury (2005) and Im-Bolter et al. (2006), who showed that school-age children with PLI had difficulties on nonlinguistic Stop-Signal and antisaccade tasks. One possible explanation is that the Go/No To task and the Incompatibility task may place less demand on response inhibition processes than StopSignal or antisaccade tasks (Johnstone et al., 2007). Therefore, school-age children with PLI may be able to perform as well as their peers with TD on less taxing tasks, but poorer on tasks that place a greater demand on inhibition processes.

\section{Updating in Preschoolers with TD}

The ability to update information depends upon the ability to hold information in mind (Garon et al., 2008). The ability to hold information in mind is important because children must be able to retain information before they can update or manipulate that information with new incoming information. Miyake et al., (2000) suggested that updating does not represent the passive ability to store task-relevant information, but rather the ability to actively manipulate relevant information in working memory.

The ability to store information without manipulating it develops earlier than the ability to update that information. This ability of storing information develops with increasing storage capacity, which is commonly assessed with memory span tasks. Memory span tasks (e.g., the forward span task) measure the maximum number of unrelated verbal items or spatial patterns that can be remembered in a correct sequence. Evidence for developmental change in verbal span shows that the number of words children retain increases from two or three words at 4 years of age to about six words at 12 years of age (Gathercole, 1998). Likewise, the numbers of blocks retained in visual 
memory span increases substantially from four blocks at age five to about 14 blocks at age 11 (Gathercole, 1998).

The ability to update and manipulate information in working memory starts to develop at three years of age and keeps increasing across preschool years into young adulthood (Best \& Miller, 2010). For example, Carlson (2005) used backward span tasks to measure updating in preschoolers from ages three to five. Relative to forward span tasks, which require remembering items in the presented sequence, backward span tasks require children to recall a sequence of items in reverse order, which requires them to actively updating and manipulate representations in mind with each incoming number. Results showed that the number of items that children remembered improved from 1.58 to 2.88 items between the ages of three to five years (Carlson, 2005; Carlson, Moses, \& Breton, 2002).

Additional evidence regarding the development of updating of working memory in young children is from the N-Back task. Tsujimoto, Kuwajima and Sawaguchi (2007) utilized visuospatial and auditory N-Back tasks to investigate working memory in two groups of children, a younger group (five- and six-year-olds) and an older group (eightand nine-year-olds). The N-Back task is a commonly used updating task in neuroimaging and behavioral studies. It requires children to determine whether the item they see/hear is the same or different from the item(s) before it. This task involves temporary storage and continuous updating of incoming items. The first important result was evidence of significant age-related improvement. Accuracy increased significantly between the younger group and the older group. The second important result was that there was a significant correlation between scores on the visuospatial and auditory N-Back tasks in 
the younger group. In contrast, the older group did not show a significant intercorrelation between these two tasks. These findings suggest that younger children were less able to update auditory and visuospatial information than older children and the modalities gradually fractionated with development.

\section{Updating in School-Age Children with PLI.}

Only one study has investigated updating in school-age children with PLI to date. Im-Bolter et al. (2006) used a visual N-Back task to test nonverbal updating ability in school-age children with and without PLI. The visual stimuli that they used were nine different configurations of three dots, which have no easily associated semantic label. Children were asked to decide whether or not each visual stimulus matched the stimulus showed in an earlier item in the sequence ( $n$ was from 0 to 2 ). The 2-back was the most complex task because it required children to monitor and hold in mind three ordered stimulus patterns that would need to be continuously updated. Results revealed that the two groups had similar performance in the 0 - and 2-back conditions. Both groups performed well in 0-back tasks, but performed at the chance level in the 2-back condition, which indicates that the 2-back was hard for both groups. However, in the 1-back condition children with PLI identified significantly fewer targets correctly than children with TD. This suggests that children with PLI had trouble updating the visual contents of working memory under conditions of moderate memory load.

\section{Mental Set Shifting in Preschoolers with TD}

In mental-set shifting tasks children must: (a) form an initial mental set in which an association is made between a specific stimulus and response in working memory; then (b) shift to a new mental set in the face of proactive interference (Garon et al., 2008). 
Some researchers suggest that mental set shifting is a relatively advanced executive function compare to inhibition and updating. These two phases of mental set shifting are thought to build upon inhibition and updating. For example, Garon et al. (2008) suggested that before children can successfully shift between two or more mental sets, they must be able to not only update and maintain the current mental set in working memory, but also to inhibit prior activated mental sets. As a result, mental set shifting may have a more protracted developmental curve than inhibition and updating (Espy, Kaufmann, McDiarmin, \& Glisky, 2001; Garon et al., 2008; Diamond, 2006).

Mental set shifting ability in preschoolers has been studied using the DCCS (Frye et al., 1995) that requires children to sort cards according to one dimension (e.g., color or pattern of the stimuli). In the pre-switch condition children were asked to sort test cards (e.g., a red rabbit or a blue ship) into two boxes marked with target cards (e.g., a blue rabbit and a red ship) by their color. In the post-switch condition children were asked to sort the same set of cards by the shape. Generally most three- to four-year-olds could sort the stimulus cards according to the first rule in the pre-switch condition, but they could not shift to the other rule during the post-switch condition. That is, they perseverated on the pre-switch rule and kept sorting the stimulus cards according to the first rule (Zelazo et al., 1996; Zelazo et al., 2003). After age four, more children could shift to the second rule in the post-switch condition (Zelazo et al., 2003; Kloo \& Perner, 2005). The accuracy of responses in the post-switch condition improved significantly from age three to five. Interestingly, although most three- and four-year-olds had a hard time shifting to the new rule in the post-switch condition, they could answer a standard verbal question about the new rule (e.g., where do blue things go in the color game?) (Zelazo et al., 1996; 
Zelazo et al., 2003). That is, children knew the current rule, but they could not execute the behavior when they sort the cards.

The three theoretical frameworks of executive function in preschoolers that were introduced in the previous sections attempt to explain why young children fail to overcome the conflict and show the perseveration. Based on the first framework, Cognitive Complexity and Control theory (Zelazo \& Frye, 1998), which views executive function as a hierarchical rule structure, there are two lower order rules underlying the higher order rule of "color" (e.g., "if red, put here", "if blue, put here") and two lower order rules under the higher order rule of "pattern" (e.g., "if it is a rabbit, put here", "if it is a ship, put here"). The explanation of why three-year-olds fail the task is that even though they know both pairs of rules embedded in the higher level rules, their hierarchical rule structures are not complex enough to integrate these two higher order rules into pairs. Therefore, they can sort the cards by both rules correctly if the rule is presented alone, but they perseverate when they fail to reflect on these higher order rule pairs in relation to one another. Thus, the two pairs of higher order rules remain unintegrated. As a result, they are not able to shift between the rules of color and pattern. According to the second framework, the Graded Representation framework (Munakata, 2001), which suggests that representations are graded and there is competition between latent and active representations, perseveration occurs when an active representation of the current rule is not strong enough to overcome a latent bias established by a previous rule. That is, perseveration occurs when there is a conflict between active and latent representations and active representation is weaker than latent representation. The reason young children fail to shift in the Dimensional Change Card 
Sort paradigm is because their active representation of the current rule is not strong enough to overcome the latent representation of the previous rule. Instead of attributing the shifting to the improvements in inhibition, Munakata et al. (2003) argued that children's successful shifting in DCCS task results from improvements in actively maintaining information, which supports relevant representations, and through interacting with inhibitory processes leads irrelevant representations to be less active. By this account, the relative strengths of active and latent representations alone can account for children's performance in shifting task (Morton \& Munakata, 2002).

Based on the third framework, Multifactoral Framework (Diamond, 2006), which suggests that there are three executive functions in preschoolers, the Diamond and Kirkham (2005) used the term "attentional inertia" to explain why three-year-olds focus on sorting the cards by the first rule but cannot shift their attention. They attribute this to immature inhibition. They hypothesize that three-year-olds have difficulty inhibiting their initial mindset (the way of thinking about the stimuli) that is no longer relevant when they have to sort the cards by the other dimension. That is, once their attention has focused on one dimension, their attention stays in that dimension, which makes it difficult to switch to the other dimension (Diamond, Carlson, \& Beck, 2005; Diamond \& Kirkham, 2005). Therefore, inhibition is needed to switch from sorting by one dimension to the other. Accordingly, effective shifting is based on how quickly and efficiently one can inhibit one's previous perspective and previous stimulus-response mappings. The above assumption was supported by Diamond, Carlson, and Beck (2005). They used the DCCS task with separate dimensions of the stimuli (e.g., a black truck on a blue background). The separated-dimension stimuli decreased the inhibition demand by never 
requiring children to inhibit the initial way of thinking about the objects pictured on the stimulus cards. For example, the truck is always a truck, therefore, children did not have to think of it sometimes as a truck and sometimes as a blue thing, which they were required to do in the standard DCCS paradigm (e.g., a blue truck). Their results showed that three times as many three-year-olds were able to switch to sorting cards by a second dimension when the dimensions were separated as could successfully switch when the dimensions were integrated in the standard DCCS paradigm.

In sum, there is no convergent view of the reason why most three- and four-yearold children have difficulty shifting from one rule to another. The ability to think about one object in different ways seems crucial for preschoolers to master the Dimensional Change Card Sort task (Kloo \& Perner, 2005). However, their performance on this task is influenced by the demand of maintaining the rule and inhibiting the previous rule/way to see the stimuli that is not relevant to the current condition (Garon et al., 2008).

\section{Mental Set Shifting in Preschoolers and School-Age Children with PLI}

To date no studies have examined mental set shifting in preschoolers with PLI. There is only one study of preschoolers with PLI that used parent and teacher ratings of behavior. Parents and teachers were asked to fill out the Behavior Rating Inventory of Executive Function-Preschool Version (BRIEF-P; Gioia, Espy, \& Isquith, 2003), a rating scale designed to investigate executive behaviors including emergent metacognition, flexibility (i.e., shifting), inhibitory self-control, and overall executive composite in everyday activities. Results showed that preschoolers with PLI had significantly higher flexibility index scores, representing poorer flexibility, compared to their peers with TD. 
Findings of mental set shifting experiments in school-age children with PLI are equivocal. Most of the behavior-based studies have shown that school-age children with PLI demonstrate comparable performance to their peers on shifting tasks when language is involved. For example, Im-Bolter et al. (2006) used the Children's Trail Making Test to measure nonlinguistic shifting ability in school-age children with PLI. There were two parts to the task. In the first part children were asked to connect, in order, the randomly distributed numbered circles (1 to 15) and they were asked to connect the circles to shift between numbers and letters in the second part (i.e., 1-A-2-B, etc.). Results showed that children with PLI performed slower on both conditions and the difference between conditions was larger for the PLI group than for the TD group. But this between-group difference disappeared after differences in motor speed and visual scanning were taken into account. These results indicated that children with PLI were slow, but they were able to shift as well as their peers. These results were supported by Henry et al. (2012), who used the same task. They found the same results in their school-age children with and without PLI.

Similar to findings above on Im-Bolter et al., (2006) and Henry et al., (2012), Dibbets, Bakker, and Jolles (2005) did not find differences in behavioral results between school-age PLI and TD groups on a Switch Task for Children. They investigated brain activation in children with and without PLI (mean age 6 years 10 months) on two switching tasks: the "Day-task" and the "Night-task," each with an associated picture. Children were asked to select the correct picture from two pictures. In the nonswitch condition one stimulus was presented repeatedly for eight trials (e.g., day-day-day....or night-night-night...); in the switch condition, eight trials of day and of night were 
randomly mixed. Typically, performance in the switch condition was slower and less accurate than the non-switch condition, which is called the switch cost. Switch costs are thought to reveal a stronger engagement of executive processes, such as the inhibition of irrelevant task set, and switching to the relevant task set. Behavioral results showed that both groups exhibited switch costs; there was no between-group difference, which suggests that no specific deficit in shifting was observed in children with PLI. In contrast, fMRI results show remarkably different activation patterns between the PLI and control groups. Children with PLI recruited additional left medial and superior temporal areas, which are normally associated with language, in the switch condition. The authors suggested that one possible interpretation was that children with PLI encountered more problems with verbalization of the rules during task performance or that they had more problems with access or retrieval of task relevant information, such as during the tasks children with PLI displayed more activation of frontal and cingulate areas that are normally associated with executive function, even when the executive function demand is low (i.e., the non-switch condition). The results suggest that the task was more effortful for the PLI group and that compensatory mechanisms were required for successful performance.

Although children with PLI performed similarly to their peers with TD on shifting tasks, they exhibited difficulties with more complex shifting task. Marton (2008) used the Wisconsin Card Sort task to evaluate the switching ability of children with PLI. This task required children to identify the relevant dimensions and to develop a sorting plan to maintain in memory. Once they demonstrated correct responses the investigator changed the sorting principle without telling them. In response children had to note the change and 
form another sorting rule according to the experimenter's feedback. Results showed that children with PLI made significantly more sorting errors than their peers with TD, which indicated that they tended to perseverate on previous dimensions, perhaps due to either inflexibility or the lack of inhibition control. Owing to the complex nature of this task, which involved inhibition and updating working memory, it was hard to determine whether the children with PLI really had difficulty in mental set shifting or whether their shifting performance was affected by poor inhibition. It is important to assess mental set shifting using a task designed to place minimal demands on inhibition and/or updating working memory.

The inconsistent results on shifting tasks have also been found in nonlinguistic shifting tasks in school-age children with PLI. Im-Bolter et al., (2006) used a Set-Shifting task in a study of executive function in children with and without PLI. The computer screen presented either one (i.e., 1 or 3) or three digits (i.e., 111 or 333). Children were asked "What number?" in the first block and were asked "How many numbers?" in the second block. In the third block children shifted between these two questions every second trial. Because the task required children to respond with a 1 or a 3 , stimuli 1 and 333 were considered to be compatible (i.e., when responses for the current task was the same as the responses for the other task) and stimuli 3 and 111 were considered to be incompatible (i.e., the response would differ depending on the task). Shifting cost was evaluated by contrasting RT and errors on the non-switch trials and the switch trials in the third block. RT results showed that the PLI group performed slower than the TD group, but the shifting costs were similar for both groups. The PLI group made significantly more errors in the incompatible condition than the TD group, but there were 
no differences in the compatible condition. The authors concluded that the PLI group had difficulty inhibiting their actions when an aspect of the stimulus was incompatible with the required response.

Henry et al., (2012) found that a PLI group performed comparably to a TD group in their nonlinguistic shifting task. They used an Intra-/Extradimensional Shift task. There were two stimuli types: simple stimuli made of colored shapes and/or white lines and complex stimuli that included both features. At first, two simple stimuli were presented and children were asked to select one. Children learned which one was correct from feedback and followed a rule. Later, the second dimension, an irrelevant white line, was introduced. The intra-dimensional shift introduced new shape and lines stimuli, but the children are to respond to the shape stimuli and ignore the white line. The extradimensional shift introduced complex stimuli, children had to switch attention to the preciously irrelevant dimension, the white line, to obtain correct responses. Total errors were measured. Children with PLI performed similarly to their peers with TD on this task.

Noterdaeme et al., (2001) found the opposite results in their study that used an attention-shifting task. This task required children to continuously shift between two categories of geometric symbols (round and angular forms). Each presentation contained a variation of both geometric categories and children were asked to shift from one type of geometric category to the other after each presentation, beginning with an angular symbol as a target in the first presentation, followed by a round symbol as a target in the next presentation. RTs and the number of shifting errors were measured. Results showed that the PLI group performed significantly slower and exhibited significantly more errors than 
the TD group. Interestingly, the authors observed that children with PLI had problems of naming and labeling visual materials during the task. Although they labeled visual materials to solve the task, they were slow and inefficient. The authors hypothesized that the poor performance of the PLI group could be the result of difficulties in the ability to name and label the visual materials rather than the ability to shift. According to their hypothesis, the PLI group may not have a shifting deficit; rather, the reason that they performed poorly on the shifting task may be due to the inefficient use of language, which hindered them from labeling material for the task, even though the task itself did not involve linguistic information.

\section{Summary}

To conclude, the preschool years are critical for executive function development. Preschoolers develop greater control of their behavior in everyday life and they become better able to modulate behavior appropriately in contexts outside their home (Isquith, Gioia, \& Espy, 2004). Among inhibition, updating, and mental set shifting, the ability to inhibit irrelevant information is thought to develop early, around age two or three, but preschoolers still have difficulty with complex inhibition tasks. The ability to update the information stored in working memory is thought to depend upon the ability to store information. As a result, updating ability starts to develop from age three. Mental set shifting is thought to develop later than inhibition and updating because the processes involved in shifting are more complex. Children must be able to form an initial mental set in which an association is made between a specific stimulus and response in working memory, then shift to a new mental set in the face of proactive interference. Studies have shown that children may not be able to shift between two rules until the age of four. It is 
during this period that the development of executive functions establishes the foundation for higher cognitive processes. If preschoolers have deficits in executive function during this critical period of development, it may negatively affect their future executive function and higher cognitive process development.

To date we lack of information about executive function in preschool-age children with PLI. Results of the only experimental study we know of showed that preschoolers with PLI have difficulty inhibiting prepotent responses and irrelevant distractions (Spaulding, 2010). A questionnaire study that used the Behavior Rating Inventory of Executive function-Preschool Version showed that children with PLI were rated as exhibiting significantly poorer executive functions relative to their peers with TD (Wittke et al., 2013).

Evidence concerning school-age children with PLI is equivocal. For inhibition, most previous studies suggest that children with PLI may have inhibition deficits, regardless of whether the tasks involve linguistic or nonlinguistic information, especially when the tasks placed high demands on inhibition. However, some of the studies found that children with PLI performed as well as their peers with TD, which may be due to the low inhibition demand of the tasks. For updating, only one study has examined updating in school children with PLI by using a nonlinguistic updating task (Im-Bolter et al., 2006). The results suggested that children with PLI are impaired in updating the contents of working memory. Finally, the majority of the studies evaluating mental set shifting found no behavioral differences between the performance of school-age children with and without PLI, regardless of whether the tasks involved linguistic or nonlinguistic information. But fMRI results (Dibbets et al., 2005) suggested that children with PLI 
increased recruitment in frontal areas associated with executive function during the shifting task. This may imply that they were less efficient at recruiting appropriate neurological pathways and appeared to be taxed more easily than their TD peers.

The above findings regarding school-age children with PLI raise a question. Many of these studies used linguistically-based executive function tasks, which required children to use their linguistic knowledge to successfully complete the tasks. Therefore, language is confounded with executive function performance in these tasks. We do not know whether children with PLI performed poorly on those tasks due to their language impairment, executive function deficits, or both. Although some studies used nonlinguistic executive function tasks, no study assessed both linguistic and nonlinguistic executive function in the same groups of preschoolers.

\section{Purpose, Research Questions, and Hypotheses}

The purpose of this study was to determine whether preschool-age children with PLI showed deficits in the executive functions of inhibition, updating, or mental set shifting compared to their age-matched peers with TD when children were required to rely more on linguistic or visual information to complete the tasks successfully. The specific question was: Are there significant between-group differences for accuracy or $\mathrm{RT}$ on the executive function tasks of inhibition, updating or mental set shifting when comparing preschoolers with PLI to age-matched preschoolers with TD?

Generally, we hypothesized that if preschoolers with PLI have domain-general executive function deficits, they would show deficits on both linguistic and visual executive function tasks; if they have intact executive function, they would only show deficits on linguistic executive function tasks due to their language impairment. More 
specifically, based on previous research with school-age children with PLI, we hypothesized that (a) preschoolers with PLI would have domain-general inhibition deficits so that we expected they would perform less accurately and slower than their peers with TD on both linguistic and visual inhibition tasks; (b) preschoolers with PLI would have domain-general updating deficits so that we expected they would perform less accurately than their peers with TD on both linguistic and visual updating task; (c) preschoolers with PLI may perform similarly to the TD group on mental set shifting so that we expected they would perform with TD on both linguistic and visual mental set shifting task in terms of accuracy. 


\title{
CHAPTER 2
}

\section{MANUSCRIPT}

Executive Function in Preschoolers with Primary Language Impairment

\author{
Hui-Chun Yang \\ Arizona State University
}

\begin{abstract}
Author Note
Hui-Chun Yang, Department of Speech and Hearing Science, Arizona State University. Correspondence concerning this proposal should be addressed to Hui-Chun Yang, Department of Speech and Hearing Science, Arizona State University, Tempe, AZ85287-0102.
\end{abstract}

E-mail: hyang66@asu.edu 


\begin{abstract}
Purpose: The purpose of this study was to evaluate whether preschoolers with primary language impairment (PLI) have deficits in executive function when compared to their peers with typical development (TD) by examining inhibition, updating, and mental set shifting.
\end{abstract}

Method: Twenty-two four- and five-year-old preschoolers with PLI and 30 preschoolers with TD completed two sets of computerized executive function tasks, three that were linguistically based and three that were visually based to minimize linguistic demands. This permitted us to test the hypothesis that poor performance on executive function tasks in preschoolers with PLI results from impaired executive function rather than language impairment.

Results: The PLI group exhibited significantly lower accuracy than their peers on linguistic inhibition, linguistic and visual updating tasks, and linguistic and visual mentalset shifting tasks. The PLI group showed comparable accuracy to their peers on the visual inhibition task.

Conclusions: Results suggest that preschool-age children with PLI have domain-general executive function deficits in updating and mental-set shifting, but not inhibition deficits as measured by our tasks. Implications for early intervention are discussed. 
Executive Function in Preschoolers with Primary Language Impairment

Children with primary language impairment (PLI) are traditionally identified by their poor performance on language measures, despite having no hearing impairment, neurological impairment, or intellectual disability (Leonard, 1998). However, research also suggests that some children with PLI have difficulties in a number of cognitive domains, including executive function (Bishop \& Borbury, 2005; Henry, Messer, \& Nash, 2012; Im-Bolter, Johnson, \& Pascual-Leone, 2006; Marton, Kelmenson, \& Pinkhasova, 2007; Spaulding, 2010).

Executive function is a collection of inter-related processes responsible for purposeful, goal-directed behavior, such as inhibiting an automatic tendency, updating information held in working memory, and shifting between mental set (Anderson, 2002; Miyake et al., 2000). Executive function develops rapidly during the preschool years (Diamon, 2006; Garon, Bryson, \& Smith, 2008). It is important to study executive function in preschoolers because research suggests it plays a critical role in language development (Carlson, Mandell, \& Williams, 2004; McClelland et al., 2007), school readiness in areas such as early literacy and numeracy (Blair \& Razza, 2007; McClelland et al., 2007), and in classroom behavior (Brock, Rimm-Kaufman, Nathanson, \& Grimm, 2009).

To date the majority of studies reporting executive function deficits in children with PLI have been restricted to school-age children. Many of these studies used "linguistically-based" executive function tasks that required children to use their linguistic knowledge to respond correctly. Thus, we do not know whether children's poor performance was due to language impairment, to executive function deficits, or both. A 
few studies have used "visually-based" tasks that did not require children to use linguistic knowledge to complete tasks, but the results were inconclusive. That is, children with PLI performed significantly poorer than their peers with typical development (TD) on some visually-based tasks (Bishop \& Norbury, 2005; Henry et al., 2012; Im-Bolter et al., 2006), but they performed comparably to their peers with TD on other visually-based tasks (Noterdaeme, Amorosa, Mildenberger, Sitter, \& Minow, 2001; Henry et al., 2012; Petruccelli, Bavin, \& Bretherton, 2012). The purpose of this investigation was to determine whether children with PLI performed differently from their peers with TD on executive function tasks that primarily relied on linguistic vs. visual processing.

\section{Executive Functions}

Executive function, also called cognitive control, is an umbrella term that refers to the allocation of attentional resources involving high-level, goal-directed behavior (Anderson, 2002). It is a collection of interrelated abilities that enable individuals to modify their thoughts and actions (Friedman et al., 2006). The most widely known framework of executive function was proposed by Miyake et al. (2000). They focused on three executive functions most likely to be involved in complex executive tasks: (a) inhibition of preponent responses and resistance of external distractor interference and internal proactive interference; (b) updating and monitoring working memory representations that require individuals to dynamically monitor and code incoming information for relevance to the task at hand, and then revise the content held in working memory by replacing no longer relevant information with more relevant information; and (c) shifting between mental sets, also called task switching, that involves the ability to engage in and disengage from tasks, as well as the ability to perform a new operation in 
the face of proactive interference or negative priming. Miyake et al. (2000) found that these three executive functions were moderately correlated with one another in undergraduate college students, but they were also separable. Thus, the authors described the nature of executive function as having both unity and diversity.

\section{Executive Function in Preschoolers}

The original three-component framework proposed by Miyake et al. (2000) has been applied to preschool-age children in developmental studies with two main findings. First, the majority of studies that have focused on organization in preschoolers found a single factor framework and failed to find the three-component framework (Hughes, 1998; Senn, Espy, \& Kaufmann, 2004; Wiebe, Espy, \& Charak, 2008; Wiebe et al., 2011). These findings suggested that executive function is unitary and less fractionated in young children than in adults. Although most of the studies found a one-factor model among all of the executive function tasks, results varied with task selection and with dependent variables. Miller, Giesbrecht, Muller, Mclnerney, and Kerns (2012) reported different findings. They asked 129 children ages three to five years to complete a battery of executive function tasks that included inhibition, updating, and mental set shifting. Their confirmatory factor analyses showed a two-factor model with an inhibition component and a component that combined updating and mental set shifting. Thus, by using age-appropriate tasks a multifactoral framework of executive function in preschoolers was found.

The second main finding regarding executive function in preschoolers is that the three identified executive functions show different developmental trajectories during the preschool period (Carlson, 2005; Diamond, 2002, 2006). In a comprehensive review of 
executive function in preschoolers, Garon, Bryson, and Smith (2008) documented that there were significant age-related gains in all three executive functions during the preschool period. Inhibition and updating developed earlier than mental set shifting, which is more complex in nature and has a more protracted course of development (Garon et al., 2008).

\section{The Relationship between Executive Function and Language in Preschoolers}

Substantial evidence suggests that there is a positive relationship between executive function and language development during the preschool years (Carlson et al., 2004; Wittke, Spaulding, \& Schechtman, 2013). There is a debate regarding whether executive function fosters language development, whether language supports executive function development, or whether the relationship is reciprocal. In a study with 50 preschoolers (mean age 47 months) Hughes (1998) found significant, positive correlations between verbal ability, including receptive and expressive language abilities and inhibitory control, and between verbal ability and working memory after age was partialled out. Mazuka, Jincho, and Oishi (2009) proposed that young children's perseverance in sentence comprehension may be due to their immature executive function. For example, when five-year-old children heard "put the frog on the napkin in the box" they tended to interpret the napkin to be the goal of the action. But when the rest of the sentence "in the box" was heard, children were unable to revise their misanalysis. In this case young children seemed to show difficulty revising their misanalysis midsentence in some temporarily ambiguous sentences, which could be because of their inability to inhibit the previous statement and shift their mind to evaluate the whole sentence. 
In a study testing the importance of language on executive function, Kirkham, Cruess, and Diamond (2003) examined the impact of no verbal labeling on the standard version of the Dimensional Change Card Sorting task (DCCS; Frye, Zelazo, \& Palfai, 1995), which required children to switch between card sorting rules (e.g., color or

pattern), versus a labeled version, which required children to label the test card according to the current sorting rule. They found that the performance of shifting to the other sorting rule was significantly better in the labeled version than the standard version in three-year-olds. It may be that labeling facilitates the ability to reflect on the rule of sorting, or it may increase the amount of time that the rule remains in working memory, or it may help direct the focus of attention by scaffolding children's ability to inhibit their focus on the previous rule (Kirkham et al., 2003). Following this logic, if children have difficulties in language, their language impairment may hinder their performance on executive function tasks.

\section{Executive Function in Children with PLI}

Relatively few studies have investigated executive function in children with PLI and most studies have been restricted to school-age children. Although there are inconsistencies among the research findings, in general researchers have reported that school- and preschool-age children with PLI have difficulties with inhibition (Marton, Campanelli, Eichorn, Scheuer, \& Yoon, 2014; Marton, Campanelli, Scheuer, Yoon, \& Eichorn, 2012; Spaulding, 2010; Finneran, Francis, \& Leonard, 2009; Oram; 2003; Bishop \& Norbury, 2005) and updating (Im-Bolter et al., 2006), regardless of whether the tasks involved language or not; but school-age children with PLI performed similarly to 
their peers with TD on behavioral mental set shifting tasks (Im-Bolter et al., 2006; Dibbets, Bakker, \& Jolles, 2006; Henry et al., 2012; Beck, 2001).

Inhibition. According to Friedman and Miyake (2004), inhibition can be differentiate among three inhibition components: (a) inhibition of prepotent response, which refers to the suppression of or stopping of automatic responses in response to a stimulus; (b) resistance to distractor interference, which refers to resisting externally irrelevant stimuli; and (c) resistance to proactive interference, which refers to suppressing unwanted internal sources (memory traces) that may affect efficient information processing. Preschoolers with TD gradually become able to suppress automatic and dominant responses and to ignore irrelevant distracters (Carlson, 2005). Our present study evaluated resistance to distractor interference and indirectly tapped resistance to proactive interference.

Inhibition of prepotent responses has been investigated in school-age and preschool-age children with PLI by using inhibition tasks that involved linguistic processing. Spaulding (2010) reported that preschoolers with PLI performed significantly less accurately on go/nogo and stop-signal tasks, which suggested that preschool-age children with PLI have difficulties in inhibiting prepotent responses. Several studies have used nonlinguistic tasks to examine the inhibition of prepotent responses in school-age children with PLI, with inconsistent results. Three reported that school-age children performed similarly to their age-matched peers with TD on non-linguistic inhibition tasks (Dodwell \& Bavin, 2008; Noterdeame et al., 2001; Marton et al. 2012); however, Bishop and Norbury, (2005) and Im-Bolter et al. (2006) found that children with PLI made more errors than their age-matched peers with TD on nonlinguistic inhibition tasks. Similar 
results were found in a study showing that preschoolers with PLI selected the stimuli that they were supposed to inhibit more than their peers with TD on a visual sustained attention task (Finneran et al., 2009). Thus, it is still unclear whether children with PLI have general deficit in inhibition of prepotent responses.

Deficits in resisting distractors have been found in preschoolers with PLI. In the study by Spaulding (2010) children with PLI had difficulty inhibiting irrelevant information, regardless of whether it was visual, nonverbal auditory, or linguistic in nature. Spaulding suggested that their inefficient inhibition may have a negative impact on the use of attentional resources because they were not be able to prevent encoding of irrelevant information, to discard previously encoded (but no longer relevant) information, or both. Deficits in resisting linguistic distractors has also been found in school-age children with PLI who demonstrated longer RTs and an increased number of errors compared to age-matched peers with TD during incongruent trials on linguistic Stroop tasks (Bishop \& Norbury, 2005; Kuntz, 2012). However, this deficit was not observed on all of the linguistic Stroop tasks. Beck Mutch (2001) and Henry et al. (2012) did not find between-group difference on linguistic Stroop or Stroop-like tasks. The mixed results were also found on nonlinguistic resistance of distractor tasks in school-age children with PLI. Henry et al. (2012) found that children with PLI made significantly more errors than their peers with TD on a nonlinguistic Stroop-like task as did Marton et al. (2012), who found that school-age children with PLI took longer than age-matched and language-matched children with TD to find the matching pair of visual patterns among similar visual distractors. Conversely, Noterdeame et al. (2001) did not find between-group differences on a nonlinguistic Simon task. This could be in response to 
the level of inhibition demand or because the response modes were different among those tasks. Thus, the results of both linguistic and nonlinguistic resisting irrelevant distractor tasks are inconclusive.

Only one study has directly examined resistance of proactive interference in school-age children with PLI by using a linguistic task. Marton et al. (2014) asked children to press the key when the word presented on the screen is belong to a category (e.g., family) and not to press the key when the distract word is either not belong to that category or from the previous task (proactive distractor). They found that children with PLI had a weakness in differentiating between task-relevant and irrelevant items and suppressing irrelevant information in terms of accuracy, but this inhibition difficulty did not reflect on the RT. They further found that the more strengthening of the item's representation is, the larger negative effect showed on response accuracy of the subsequent item in children with PLI than in children with TD. These two findings revealed that school-age children with PLI demonstrated poor resistance to the distractors that had been memorized before even though they were irrelevant to the current task.

Updating. Updating is the ability to refresh and manipulate the information in working memory. According to Miyake et al. (2000), updating is actively manipulating relevant information in working memory, not only passively storing task-relevant information. Children must be able to retain the information in short term memory before they can actively update or manipulate it with incoming information (Garon et al., 2008). Generally, the number of items that preschoolers with TD are able to update increases from about 1.58 to 2.88 items between the ages of three to five years as indexed by backward span tasks (Carlson, 2005). 
Only one study to date has specifically assessed "updating" ability in school-age children with PLI. Im-Bolter et al. (2006) compared the updating ability of 45 children with PLI and 45 age-matched children with TD using a visual n-back task. The PLI group scored significantly lower than the TD group under conditions of moderate memory load, suggesting that children with PLI had trouble updating the visual contents of working memory. These results were consistent with the recent meta-analyses of visuospatial working memory in children with PLI that found significant between-group differences in visuospatial storage (effect size 0.49 ) and visuospatial central executive function tasks (effect size 0.63) in favor of the TD group, which supports the idea that the working memory deficits of children with PLI is domain-general (Vugs, Cuperus, Hendriks, \& Verhoeven., 2013).

No studies to date have specifically assessed updating in preschool-age children with PLI. Several studies have investigated working memory in preschoolers with PLI by using complex working memory tasks. For example, Petruccelli, Bavin, and Bretherton (2012) evaluated working memory in preschoolers with PLI in a multimodal context based on Baddelay's (2003) working memory model, which includes a phonological loop, visuospatial sketchpad, episodic buffer, and central executive. Their findings showed that five-year-olds with PLI only exhibited significantly poorer performance than peers with TD in phonological loop and episodic buffer functions, but not in visuospatial or central executive functions. In contrast, Vugs, Hendriks, Cuperus, and Verhoeven (2014), who examined verbal storage and central executive, visuospatial storage, and central executive functions, reported that four- and five-year-olds with PLI performed 
poorer that those with TD on all tasks, supporting the idea of domain-general deficits in working memory.

Mental set shifting (Shifting). Shifting is the ability to flexibly switch perspectives, focus of attention, or the mappings between stimulus and response (Diamond, 2006). Two phases take place: (a) forming an initial mental set in which an association is made between a specific stimulus and response in working memory; and (b) shifting to a new mental set in the face of proactive interference (Garon et al., 2008). Successful shifting requires children not only to update and maintain the current mental set in working memory, but also to inhibit previously activated mental sets (Diamond, 2006; Garon et al., 2008). This ability is often measured using the DCCS, which asks children to sort cards by one dimension (e.g., pattern) in the pre-switch condition, then sort the same cards by another dimension (e.g., color) in the post-switch condition. Zelazo, Frye, and Rapus (1996) asked 60 three-and four-year-old children with TD to complete the DCCS task and to answer a knowledge question (e.g., where do blue things go in the color game?). They found that 18 out of 30 three-year-olds failed to shift to the second dimension in the post-switch condition, whereas 27 out of 30 four-year-olds successfully switched between rules. Interestingly, of the 18 three-year-olds who failed to switch, 16 could answer knowledge questions regarding the second dimension in the post-switch condition. It appeared that the three-year-olds knew the current sorting dimension, but when they saw a stimulus relevant to both the previous and the current sorting rules, they focused attention on the previously relevant dimension, which may be due more to inefficient inhibition than to working memory (Diamond \& Kirkham, 2005). 
Most behavioral results show that school-age children with PLI exhibit comparable performance to their peers with TD on many linguistic shifting tasks (ImBolter et al., 2006; Henry et al., 2012; Dibbets et al., 2006; Beck Mutch, 2001). For example, Im-Bolter et al. (2006) used the children's trail making test to assess 45 children with PLI and 45 age-matched children with TD. Results showed that the PLI group performed more slowly and less accurately overall than the TD group, but there was no between-group difference in switch costs in terms of RT and accuracy (the difference between pre-shift and post-shit blocks), which suggests that children with PLI could shift as well as their peers. Interestingly, the error analysis showed that the PLI group made more errors in the incompatible condition than the TD group, which indicated that they did not have shifting deficits, but had difficulty inhibiting their actions when an aspect of the stimulus was incompatible with the required response. Conversely Marton (2008) found that children with PLI made more total errors and perseveration errors and had difficulty developing new sorting rules on the standardized Wisconsin card sorting task. These inconsistent results may be because of the different dependent variables used in the studies. Morton (2008) reported the total errors and perseverative errors rather than the switch costs between pre-shift and post shift blocks. Since children with PLI usually make significantly more errors or are slower than their peers with TD, subtracting the performance in the pre-shifting block from the post-shifting block may decreases the magnitude of between-group differences.

Two studies that used nonlinguistic shifting tasks also suggested that school-age children with PLI were slower and made more errors than their peers with TD, but their switch costs were similar to their peers with TD (Im-Bolter et al., 2006; Henry et al., 
2012). But these results differ from Noterdaeme et al. (2001) who reported that children with PLI performed significantly slower and made more switch errors than their peers with TD when they had to continuously shift between two categories of geometric symbols. Additionally, the authors stated that even though the stimuli themselves did not involve linguist information, children with TD were able to name and label visual materials during the task, whereas, the PLI group was inefficient in doing so. Thus, the inefficient use of language may hinder PLI group performance on nonlinguistic shifting tasks.

Summary. Preschoolers with PLI exhibit weaknesses in inhibiting prepotent responses and ignoring distractors on linguistic inhibition tasks. Mixed results have been reported for school-age children with PLI in their ability to inhibit nonlinguistic prepotent responses and to resist linguistic and nonlinguistic distracters. Poor resistance of proactive distractors has been found on linguistic inhibition tasks in school-age children with PLI. School-age children with PLI have difficulty in updating visuospatial information, which suggests that their updating deficits are domain-general. Most of the behavior-based evidence suggests that school-age children with PLI perform similarly to their peers with TD on linguistic and nonlinguistic shifting tasks. So far, we do not have information about how preschoolers with PLI perform on nonlinguistic inhibition tasks and on linguistic or nonlinguistic updating and shifting tasks.

\section{Method}

\section{Participants}

Two groups of four- and five year-old preschoolers completed this study: 22 children with PLI (mean age $=56.77$ months) and 30 children with TD (mean age $=57.3$ 
months). There were 25 boys and 27 girls. Twelve children were Hispanic and 40 were Non-Hispanic. One child was Native American, two were Asian, three were African American, and 36 were White. Eight children were more than one race and two did not report.

All of the participants were recruited from public and private preschools and daycares in the Phoenix metropolitan area. Parents were asked to complete a questionnaire regarding the mother's education level. The questionnaire also included 18 items from the Strengths and Weaknesses of ADHD Symptoms and Normal Behavior Scale (SWAN; Swanson et al., 2005), which asks about children's attention and impulse control. As described below in exclusionary criteria, we excluded one child in the TD group and two children with PLI who were rated extremely high on this scale. Since there was a significant difference between the two groups on this measure (mean score for the PLI group was 8.23 and for TD group was 3.14) and because poor attentional control can affect performance on executive function tasks, the raw score on this questionnaire was included as a covariate in the data analyses. According to parent report, all of the children spoke English as their primary language.

All children met the following criteria: (a) demonstrated normal hearing sensitivity by passing a pure-tone hearing screening at $25 \mathrm{~dB}$ HL at 500, 1000, 2000, and $4000 \mathrm{~Hz}$ in each ear; (b) no history of neuropsychiatric disorders (e.g., ADD/ADHD, emotional disability, motor disorder) by parent report; and (c) standard score of 75 or higher on the nonverbal matrices subtest of the Kaufman Brief Intelligence Test - Second Edition (K-BIT-2, Kaufman, \& Kaufman, 2004). To be included in the PLI group, children were required to score 87 or lower on the Structured Photographic Expressive 
Language Test-Preschool Second Edition (SPELT-P2; Dawson, Eyer, Fonkalsrud, 2005).

This cutoff score is based on the discriminant analysis from Greenslade, Plante, and Vance (2009) that yielded $90.6 \%$ sensitivity and 100\% specificity for the SPELT-P2 in identifying preschoolers with language impairment. To be included in the TD group, children were required to score 95 or higher on the SPELT-P2. Descriptive information about children and their performance on inclusionary measures is presented in Table 2 .

\section{General Procedures}

Children were tested individually over two, 90-minute sessions on separate days, with a 10-minute break within each session. In the first session children completed the eligibility assessments, including the hearing screening, K-BIT, and SPELT-P2, with tests administered in counterbalanced order across groups. In the second session the two baseline RT tasks and six executive function tasks were administered to children on a Dell laptop computer equipped with DMDX software (Forster \& Forster, 2003). Children were seated next to a trained research assistant (RA), who supervised their play. Children and RAs wore headphones and children's headsets also had an attached microphone. Children responded to the tasks by either pressing a marked key on the computer keyboard or by voice recorded by DMDX software. Before starting the experimental tasks, children were instructed to place their dominant hand on a large green circle taped to the table in front of the, located a standard distance from the response key.

\section{Experimental Tasks}

Six experimental executive function tasks and two baseline RT tasks were administered in a counterbalanced order across children. The two baseline RT tasks were to document children's motor RTs when there was minimal language or cognitive 
demand because studies have shown that children with PLI are slower than their peers with TD on motor responses (Miller, Kail, Leonard, \& Tomblin, 2001; Schul, Stiles, Wulfeck, \& Townsend, 2004). The three executive functions of inhibition, updating, and mental set shifting were assessed using two computerized tasks, one linguistically-based task and one visually-based task. Tasks were designed to avoid floor or ceiling effects for preschoolers with and without PLI. Each task started with recorded instructions, followed by a training phase that taught children how to play the game. Children received feedback regarding the accuracy of their responses during training. A range of four to eight practice trials were given after the training phase, which children could repeat up to three times before being disqualified from that task. Please see Table 3 for the description and the disqualification criteria for each executive function task.

Motor RT task. This task assessed children's motor RTs for a key press when a visual image of a soccer ball appeared on the screen. One image was presented at the center of the screen at a time. Children were asked to press the right shift key (with a white circle sticker on it) as fast as they could when they saw the soccer ball. Children had to respond correctly to 3 out of 4 trials to pass the training. The testing phase began with a $500 \mathrm{~ms}$ fixation cross on the screen. It included 20 trials with 10 target trials (image was presented) and 10 non-target trials (blank screen presented) in a random order. Each image was presented for $1500 \mathrm{~ms}$. The next stimulus appeared $500 \mathrm{~ms}$ after the response. If the child did not respond after $2500 \mathrm{~ms}$ the program presented the next stimulus. The program recorded the accuracy and the RT in ms from the onset of the picture stimulus to the key-press response. The dependent variable was the mean RT of correct target trials. 
Voice RT task. This task assessed children's voice onset times when they said the word 'cake' in response to seeing the image of a cake appear on the screen. Cake was selected because the $/ \mathrm{k} /$ phoneme was also found in other stimuli in tasks requiring a verbal response. The procedure was the same as the key press RT task, except that children were asked to say "cake" as fast as they could when they saw the cake image. The program recorded children's voice responses. The researcher assessed the accuracy of responses and calculated the RTs of children's verbal responses in the lab using voice recordings. The dependent variable was the mean RT for all correct target trials.

Inhibition tasks. The linguistic inhibition task used a child version of the flanker task adapted from Rueda, Posner, Rothbart, and Davis-Stober (2004). It measured children's ability to focus on the target while inhibiting the distractions. See Figure 1 for an illustration. One horizontal row of five animals was presented in the center of the screen at a time. The middle animal was the target and the surrounding animals were the flankers. There were two conditions: congruent, where all five animals were the same (either all cats or dogs), and incongruent, where the animals surrounding the middle animal were different (i.e., a dog was surrounded by four cats or a cat was surrounded by four dogs). The congruent flankers facilitated the correct response and incongruent flankers interfered with the correct response (Verbruggen, Liefooghe, \& Vandierendonck, 2004). Children were asked to play hide-and-seek with the middle animals. They were told they would find them by saying 'cat' or 'dog' that matched the middle animal. Each trial began with a $500 \mathrm{~ms}$ fixation cross on the screen followed by a 10 second stimulus presentation. The next stimulus appeared $1000 \mathrm{~ms}$ after the response. The task included 32 test trials (16 congruent, 16 incongruent) presented in a random order by the 
computer. RAs assessed the accuracy of responses and calculated the RTs of children's verbal responses in the lab using voice recordings that were recorded by the program. There were two dependent variables. The first was the conflict effect for accuracy, which refers to the difference in percentage correct between congruent and incongruent conditions. This indexes the inhibitory effort that children have to make during the incongruent trials. It is calculated by subtracting the mean percent correct in the congruent condition from the mean of percent correct in the incongruent condition. The second dependent variable was the conflict effect for RT which is the mean RT difference for accurate trials in the incongruent condition minus the mean RT for correct trials in the congruent condition.

The visual inhibition task used the child version of the flanker task developed by Rueda et al., (2004). See Figure 1 for an illustration. The stimuli were selected from stimuli at the Department of Kinesiology at Michigan State University (http://education.msu.edu/kin/hbcl/software.html). In this task a horizontal row of five fish was presented in the center of the screen. The middle fish was the target and the surrounding fish were the flankers. There were two conditions: congruent, where all fish pointed in the same direction (either right or left), and incongruent, where the fish surrounding the middle fish pointed in the opposite direction of the target fish (either right or left). Children were told that the middle fish was hungry and they could feed it by pressing the key that matched the direction it was pointing. Each test trial began with a $500 \mathrm{~ms}$ fixation cross on the screen followed by a stimulus presentation. If the children did not respond, the stimulus would stay on the screen for 10 seconds. After children respond or after the 10 seconds without response, a $1000 \mathrm{~ms}$ intra-stimulus-interval was 
presented with blank screen. The task included 32 trials (16 congruent, 16 incongruent) presented randomly by the computer. Accuracy and RTs, measured in ms from the onset of the test stimulus to the key press, were recorded by DMDX software. The dependent variables were the same as the linguistic inhibition task.

Updating tasks. The linguistic updating task used a visual n-back task adapted from Im-Bolter et al. (2006). It measured children's ability to continually update the contents of working memory. To successfully complete the task children were required to compare each incoming image with the one just before it and to disregard the images once they were more than 1-back. See Figure 1 for an illustration. The task used eight images (car, duck, horse, apple, chair, shoe, flower, and hat). Words used in the task were selected from four semantic categories of the MacArthur Communicative Development Inventory (Fenson et al., 1994) as names known by $70 \%$ or more of children under three years of age. A single image was presented at the center of the screen. Children were asked to help match toys by saying "same" when the toy was the same as the one on the previous screen (same trial) and saying "different" if it was different from the one on the previous screen (different trial). The testing phase began with a $500 \mathrm{~ms}$ fixation cross on the screen. Each stimulus was presented for $3000 \mathrm{~ms}$, followed by a $2000 \mathrm{~ms}$ blank screen. The task included 36 trials (18 same trials and 18 different trials) presented in a fixed order for each child. The researcher assessed the accuracy of children's verbal responses in the lab using voice recordings that were recorded by the program. The dependent variable was accuracy calculated as the percentage of correct responses.

The visual updating task used a visuospatial n-back task adapted from Carlson et al. (1998). See Figure 1 for an illustration. In this task a cartoon butterfly was presented 
on the screen in one of eight locations. Children were asked to say "stayed" if the butterfly stayed at the same location as the previous screen (stay trial) or say "moved" if the butterfly moved to a different location from the previous screen (move trial). All other parameters and dependent variables in the testing phases were analogous to the linguistic updating task.

Shifting tasks. The linguistic shifting task utilized the separate-dimensions version of the DCCS task (Diamond, Carlson, \& Beck, 2005). It measured children's ability to shift between two sorting rules. The original DCCS task used colored shapes. Because four-year-old children may still not have mastered the ability to sort one item using two different dimensions (e.g., "blue rabbit" can be considered as "blue" or as a "rabbit"), they need more inhibition to suppress the first dimension when they need to sort the stimulus by the second dimension. In the separate-dimension version of the task each stimulus had two separate dimensions. One was the color of the background and the other one was the shape of the item (e.g., black glasses presented on a red background). The separate-dimension design decreases the degree of inhibition required to successfully complete the task because the shapes and colors are separate. There were two target stimuli and two test stimuli. The target stimuli included a pair of black glasses on a red background and a pair of black scissors on a blue background. These target stimuli were always fixed at the bottom of screen. The test stimuli were a pair of black glasses on a blue background and a pair of black scissors on a red background that were randomly presented in the middle of the screen each time. Children saw a single test stimulus presented in the middle of the screen and two target stimuli fixed on the bottom right and left of the screen. Children were asked to sort each test stimulus either by the color of the 
background or by the shape. The order of sorting by color and shape was counterbalanced across children.

This task contained one pre-shift block and one post-shift block, with six trials per block. Before each block, children were reminded of rules for the current sorting game and asked to demonstrated knowledge of both rules for the current game by pointing to the appropriate target stimuli in answer to the knowledge questions (e.g., where do the blue ones go in the color game?). The testing phase began with a $500 \mathrm{~ms}$ fixation cross on the screen. The test stimuli was presented on the screen. Simultaneously, children heard auditory instructions that indicated the current sorting dimension of the stimulus (e.g., this is a blue card, where does it go?), which reduced the working memory demand of remembering the sorting rule. If the child did not respond, the stimulus remained on the screen for 10 seconds. After children responded or after 10 seconds without response, a $1000 \mathrm{~ms}$ intra-stimulus-interval was presented, with only the target stimuli on the screen. The dependent variable was accuracy, calculated by the percentage of correct responses in the post-shifting block. The reason that we only used accuracy in the post-shifting block as the dependent variable was because children with TD typically show a ceiling effect in the pre-shifting trials (Zelazo, Muller, Frye, \& Marcovitch, 2003), which was also the case in our study (the mean of percent of correct responses was $99 \%$ for the TD group). Further, scores on the post-shifting block reflect children ability to shift their mental-set.

The visual shifting task used the same paradigm as the linguistic shifting task; however, the target and test stimuli did not have readily associated names. See the bottom of Figure 1 for an illustration. Each stimulus had two separate dimensions; one was the 
pattern of the background and the other was the shape of a white polygon. The two target stimuli were different polygon shapes presented on backgrounds of two different patterns (diagonal-lined or tessellated pattern). As shown in Figure 1, if children were asked to sort by background pattern they would have to press the right key and if children were asked to sort the same test stimulus by the shape they would have to press the left key.

Internal consistency estimates of reliability for each task. The internal consistency for each task was assessed by calculating split-half reliability coefficients expressed as Spearman-Brown corrected correlations. Results of the split-half reliability coefficients for each task are reported in Table 4 . We followed three steps to compute the split-half coefficient for the TD group, the PLI group, and the combined groups. First, we split each task into two halves, which were treated as two split scores; second, we computed the correlation between the two split scores; finally, we computed the reliability by using the Spearman-Brown formula.

We obtained splits for each task so that they were as equivalent as possible given the different characteristics of each task. For the two baseline RT tasks, RT means for correct responses were computed separately for the odd-numbered and the evennumbered trials (i.e., $S_{\text {odd }}, S_{\text {even }}$ ). For the two inhibition tasks the differences in accuracy and RT means for correct responses between the incongruent and congruent trials were the dependent variables. We applied the following two steps to split the data. First, we computed the means of percent correct and the RTs of correct responses for the oddnumbered congruent trials, the even-numbered congruent trials, the odd-numbered incongruent trials, and the even-numbered incongruent trials separately (e.g., $\mathrm{S}_{\text {congruent_odd, }}$ $\left.\mathrm{S}_{\text {congruent_even, }}, \mathrm{S}_{\text {incongruent_odd, }}, \mathrm{S}_{\text {incongruent_even }}\right)$. We also calculated and reported the split-half 
coefficients for the congruent and incongruent trials separately. Second, we computed the difference between the odd-numbered congruent trials and the odd-number incongruent trials, as well as the difference between the even-numbered congruent trials and the evennumbered trials for accuracy and RT (e.g., $S_{\text {congruent_odd }}-S_{\text {incongruent_odd }}=S_{\text {difference_odd, }}$ $\left.S_{\text {congruent_even }}-S_{\text {incongruent_even }}=S_{\text {difference_even }}\right)$. The differences for odd-numbered trials and even-numbered trials were our split scores (e.g., $\left.\mathrm{S}_{\text {difference_odd, }}, \mathrm{S}_{\text {difference_even }}\right)$. For the two updating tasks, mean accuracy for all the trials was the dependent variable. The mean percent correct was computed separately for the odd-numbered and the even-numbered trials. For the two shifting tasks mean accuracy for all the post-shifting trials was the dependent variable. The mean percent correct was computed separately for the oddnumbered and the even-numbered trials. These two splits of each task were then treated as split scores to calculate a split-half coefficient.

According to Kline (2000) a cut-off of .7 is suitable for cognitive tests and values below .7 can be expected because of the diversity of the psychological constructs being measured. All of our computerized tasks achieved acceptable reliability of .7 or better when both groups were combined, except for the difference scores for accuracy and RT on the linguistic inhibition task and difference scores for RTs on the visual inhibition task. Note however that the reliability of difference scores can be low due using two highly correlated scores to calculate the difference scores, which is the case for our scores on the inhibition task. For the linguistic inhibition task, the congruent and incongruent trials were significantly correlated for accuracy, .53, and RT, .76; for the visual inhibition task the congruent and incongruent trials were significantly correlated for accuracy, .56, and RT, .88. 


\section{Statistical Analyses}

Data trimming procedure. RT data was trimmed individually by deleting any RT faster than $200 \mathrm{~ms}$ because it was not possible for children to process the stimulus and to respond within this short time frame (Eckner, Kutcher, \& Richardson, 2010). Less than $1 \%$ of the data were eliminated on the basis of this procedure.

Between-group difference analyses. To determine whether there was a significant between-group difference on each task we conducted a series analyses of variance (ANOVAs), one for each task. The between-group factor was group. Because scores on the attention rating scale were significantly higher for the PLI than TD group and because attention could impact performance on our tasks, we also conducted a series of analyses of covariance (ANCOVAs) to test whether any between-group differences remained after controlling for attention scale scores. Although mother's education level also differed significantly between groups, we did not include it as a covariate because we thought it unlikely that the one year difference would have a practical effect on the outcome of the experimental tasks. We also tested whether there was a significant difference in baseline task scores after controlling for scores on the attention scale and if so, included baseline scores as an additional covariate in analyses. Before conducting each ANCOVA analysis a preliminary analysis was conducted to evaluate the homogeneity-of-slopes assumption. All of our preliminary analyses confirmed that the relationship between the covariate(s) and the dependent variable did not differ significantly as a function of the independent variable. Effect size calculations were based on Cohen's (1988) categories: small effect size: $d=0.2$, medium: $d=0.5$, and large: $d=0.8$. 


\section{Results}

The estimated marginal means, $F$ tests, and $d$ values for the ANOVAs are reported in Table 5 and the results for the ANCOVAs are reported in Table 6. We report the ANOVA results in Table 5 for readers who are interested in whether there was a change when covariate(s) were included, but report only ANCOVA results below in the text.

\section{Baseline RT Tasks}

All children passed training for the motor and voice RT tasks. Data for the voice RT task was missing for one child from the PLI group, so this child was excluded from this analysis for the voice RT tasks. For the motor RT task the ANCOVA was significant, $F(1,49)=8.87, p=.004, d=1.03$, with the PLI group responding significantly slower than the TD group. The attention score was not a significant covariate, $F(1,49)=.02, p=.887$. Based on these findings, motor RT was included as a covariate in the analyses for visual inhibition tasks that utilized motor responses. For the voice RT task, group differences were not significant after covarying attention scores, $F(1,48)=1.61, p=.21, d=.44$. Attention scores were a significant covariate, $F(1,48)=6.95, p=.011$. Because there were no significant between-group differences, voice RTs were not included as a covariate in the analyses for the linguistic inhibition tasks where RTs were the dependent variable.

\section{Inhibition Tasks}

All children in the TD group passed training for the linguistic and visual inhibition tasks. The pass rate was only slightly lower for the PLI group, with $95 \%$ passing the linguistic training and $100 \%$ the visual training. Prior to testing for betweengroup differences, we tested for conflict effects for both groups on both tasks separately. 
Theoretically, a conflict effect occurs when individuals perform more slowly and make more errors for the incongruent than the congruent trials on the flanker task because it requires individuals to inhibit irrelevant flankers. It was important to test whether each group showed the conflict effect for the linguistic and visual inhibition tasks because if they did not demonstrate a conflict effect, this would suggest that they did not use inhibition to complete the task and that the tasks were not sensitive to inhibition. For the linguistic inhibition task four paired-samples $t$ tests were conducted to determine whether there was a conflict effect, one for each dependent variable (accuracy and RTs for congruent and incongruent trials) for each group (TD, PLI). Results showed significant conflict effects for both accuracy and RTs for the TD group $t(29)=2.25, p=.032, d=.41$ and $t(29)=4.17, p<.001, d=.76$ respectively, and for the PLI group $t(20)=3.13, p=.005$, $d=.68$ and $t(20)=3.26, p<.004, d=.71$ respectively. For the visual inhibition task the same four paired-samples $t$-tests were conducted to determine whether there was a conflict effect. Conflict effects were found for accuracy and RTs in the TD group $t(29)=2.57$, $p=.015, d=.47$ and $t(29)=4.12, p<.001, d=.75$ respectively and for the PLI group $t(21)=2.45, p=.024, d=.53$ and $t(21)=5.24, p<.001, d=1.14$ respectively. These results showed that both groups made more errors and responded slower to incongruent stimuli than congruent stimuli on both inhibition tasks.

To test for between-group differences for the conflict effect for the linguistic inhibition task we conducted two separate one-way ANCOVAs with attention scores as a covariate, one for accuracy and one for RTs. Results showed a significant between-group difference for accuracy $F(1,48)=4.27, p=.044, d=.73$, but attention scores were not a significant covariate, $F(1,48)=.38, p=.543)$. There was no between-group difference for 
RTs $F(1,48)=.75, p=.39, d=.30$. In addition, attention scores were not a significant covariate $F(1,48)=.71, p=.404$. Thus, the PLI group showed a significantly larger conflict effect than the TD group for accuracy on the linguistic inhibition task, but RTs did not differ by group.

Two one-way ANCOVAs were conducted to test for between-group differences for the conflict effect for the visual inhibition task, one for accuracy with attention scores as a covariate and one for RTs with attention scores and motor RTs as covariates. Results showed no between-group differences for accuracy $F(1,49)=.86, p=.36, d=.32$ and attention scores were not a significant covariate, $F(1,49)=.11, p=.74)$. There were no between-group differences for RTs $F(1,48)=.43, p=.517, d=.24$. Neither attention scores $F(1,48)=.19, p=.667$ or motor RT scores $F(1,48)=.20, p=.656)$ were a significant covariate. Therefore, the PLI and TD groups did not differ on either accuracy or RTs on the visual inhibition task.

\section{Updating Tasks}

The rates for passing training on the linguistic and visual updating tasks for the TD groups were both $100 \%$ and were $68.2 \%$ and $81.8 \%$, respectively, for the PLI group. Seven children with PLI could not pass training for the linguistic updating task after repeating it three times. Four of those seven children also did not pass training for the visual updating task. To evaluate whether the proportion of children who failed the training trials differed by group, two separate two-way contingency table analyses were conducted, one for the linguistic updating task and one for the visual updating task. The variables for each analysis were group (TD, PLI) and training performance (pass, not pass). Group and training performance were significantly related for the linguistic 
updating task Pearson $\chi^{2}(1, N=52)=11.03, p<.001$, Cramer's $V=.46$ (medium effect size) and for the visual updating task, Pearson $\chi^{2}(1, N=52)=5.91, p=.015$, Cramer's $V=$ .34 (medium effect size), showing that the proportion of the children who failed the training trials was significantly larger in the PLI group than the TD group on both updating tasks. Results indicate that the updating tasks were more difficult for the PLI group than the TD group, especially the linguistic task. These results must be considered in conjunction with PLI performance for those children who passed training and completed the tasks.

Because a large proportion of children with PLI failed to pass the training trials for the linguistic updating task, we also conducted an independent-sample $t$ test to confirm that the remained children in the two groups did not differ by age $t(43)=-.432$, $p=.668, d=.14$ and they did not; therefore, we proceeded with the planned ANCOVA analysis. For the children who passed training and completed the tasks, two separated one-way ANCOVAs were used to test between-group differences for accuracy, one for linguistic updating and one for visual updating. ANCOVA results showed significant between-group differences on both the linguistic updating task $F(1,42)=9.02, p=.004$, $d=1.23$, where attention scores were not a significant covariate, $F(1,42)<.001, p=.984)$, and for the visual updating task $F(1,45)=7.06, p=.011, d=1.30$, where attention scores were not a significant covariate $F(1,45)=.45, p=.504$. Thus, the PLI group made significantly more errors than the TD group on both updating tasks.

\section{Shifting Tasks}

The training pass rates for the linguistic and visual shifting tasks were $100 \%$ for both the TD and PLI groups. We also analyzed whether both groups were able to 
understand the sorting rules before they encountered the pre-shifting and post-shifting blocks by asking them to demonstrate knowledge of sorting rules for the current dimension. We conducted two one-way ANCOVAs with the percentage of correct responses on knowledge questions about the sorting rules as the dependent variable and group as the between-group variable, one for the linguistic shifting task and one for the visual updating task. Differences for the linguistic shifting task were not significant, $F(1,49)=1.23, p=.273, d=.38$ and attention scores were not a significant covariate $F(1,49)=.13, p=.719$. Differences for the visual shifting task were also not significant $F(1,49)=2.13, p=.151, d=.50$ and attention scores were not a significant covariate $F(1,49)=2.88, p=.096$. These results suggest that the groups did not differ in their understanding of the sorting rules.

However, even when all of the children demonstrated their understanding of the sorting rules before each block, many children were not able to sort the stimuli according to the correct rules during the pre-shifting block, and especially children in the PLI group. Being able to sort was defined as sorting stimuli correctly above the $50 \%$ chance level. Two children with TD (6.7\%) and three children with PLI (13.6\%) could not sort the stimuli during the linguistic pre-shift block. Two different children with TD (6.7\%) and 10 children with PLI (45.5\%) could not sort correctly during the visual pre-shift block. This showed that a large proportion of the PLI group had difficulty with the visual shifting task, even after they demonstrated sufficient knowledge of the dimensions of the stimuli and the sorting rules. Most of these children with PLI appeared stuck in the "shape" dimension, although they recognized the "pattern" dimension in training for visual shifting task. A two-way contingency table analysis was conducted to evaluate 
whether the proportion of children who failed the visual pre-shifting block were the same for both groups. Variables were group (PLI, TD) and visual pre-shifting block performance (pass, not pass). Group and visual pre-shifting block performance were found to be significantly related, Pearson $\chi^{2}(1, N=52)=10.76, p<.001$, Cramer's $V=.46$ (medium effect size), showing that the proportion of the children in the PLI group who failed the visual pre-shifting block was significantly larger than the proportion of children in the TD group. Children who could not complete the pre-shifting block above chance level were excluded from further analyses because they were unable to follow the instructions to sorting the stimuli by the required rules; therefore, they would not be able to shift rules for the post-shifting block. We conducted an independent-sample $t$ test to confirm that the remaining children in the two groups did not differ by age $t(38)=-.065$, $p=.949, d=.02$ and they did not; therefore, we proceeded with the ANCOVA.

Results for the remaining 47 children (28 TD, 19 PLI) were analyzed for accuracy in the linguistic post-shifting block and results for 40 children (28 TD, 12 PLI) were analyzed for their accuracy in the visual post-shifting block using separate ANCOVAs. Results for the linguistic post-shifting block showed significant between-group differences, $F(1,44)=5.66, p=.022, d=.95$ but attention scores were not a significant covariate $F(1,44)=.07, p=.795$, showing that the PLI group was significantly less accurate on the linguistic post-shifting block than the TD group. There was no between-group difference for the visual post-shifting block, $F(1,37)=2.81, p=.102, d=.77$ and attention scores were not a significant covariate $F(1,37)=.38, p=.541$; however, there was a medium between-group effect size with the adjusted means for the percentage of correct responses at $57.4 \%$ for the TD group and $28.6 \%$ for the PLI group. These results show 
that on average, the TD group exceeded the chance level, but the PLI group was below chance level, indicated that children in the PLI group continued to sort the stimuli by the first dimension rather than shifting. Thus, statistically the PLI group made significantly more errors than the TD group on linguistic post-shifting block, but not on visual postshifting block.

\section{Discussion}

The purpose of this study was to determine whether children with PLI performed differently from their peers with TD on executive function tasks that primarily relied on linguistic vs. visual processing. We hypothesized that if preschoolers with PLI have general executive function deficits, they would show deficits on both linguistic and visual executive function tasks; if they have intact executive function, they would only show deficits on linguistic executive function tasks, but not on visual task. Our study controlled for the potential confounding factor of an attention deficit by excluding children with PLI who were diagnosed with ADHD or who had high ratings on an ADHD scale. Further, we used scores on an attention rating scale as a covariate in our analyses.

\section{Inhibition}

Based on previous research we hypothesized that preschoolers with PLI would exhibit a general inhibition deficit; therefore, it would be difficult for them to inhibit irrelevant information presented during an inhibition task; however, our results indicated that preschool-age children with PLI performed less accurately on the linguistic inhibition task, but not on the visual inhibition task. In addition, both groups exhibited similar RTs on the two inhibition tasks. These results suggest that the PLI group did not 
demonstrate a general inhibition deficit, making it more plausible that language impairment negatively impacted their performance on the linguistic inhibition task.

The PLI group made more errors when they had to resist and inhibit surrounding linguistic distractors on our linguistic version of the flanker task. Previous studies have shown that school-age children with PLI have difficulty inhibiting irrelevant linguistic information (Bishop \& Norbury, 2005; Kuntz, 2012). Further, results from the only study of preschool-age children with PLI demonstrated that children performed significantly less accurately than their peers with TD when they had to inhibit and resist irrelevant linguistic distractors (Spaulding, 2010). To complete our flanker task, children had to first recognize and evaluate the conflict between target and surrounding distractors and then realize that they needed to execute a specific action in this conflict situation. Children then had to inhibit and suppress the irrelevant distractors to respond appropriately in a goal-directed manner. Additionally, our linguistic inhibition task placed demands on the linguistic system. The stimuli were linguistic and had a semantic relation between the to-be-inhibited and correct responses (i.e., "dog" and "cat" are semantically related). Both of the semantic representations were activated and held in an activated state during the task (Simpson et al., 2012). This task required children to inhibit the semantically-related representation in order to correctly respond to the target, which may have been difficult for the PLI group because of their weakness in processing and inhibiting semantically-related information (Cummings \& Ceponiene, 2010).

The weakness in processing and inhibiting semantically-related information in children with PLI has also been demonstrated when children were asked to name pictures while ignoring auditory distractors that were semantically-related (Seiger-Gardner \& 
Schwartz, 2008). Children with PLI responded slower to the target pictures, even when the presence of semantic interference words was $300 \mathrm{~ms}$ later than target, whereas adults and TD group revealed no semantic inhibition effect at the late $300 \mathrm{~ms}$. The authors suggested that the slow processing speed for semantic information by children with PLI may be because of a slow decay rate of semantic alternative representations in the lexical system, which interferes with later semantic processing. In our task, the slow decay rate of semantic representations in children with PLI could keep the semantically-related representations of the distractors active in working memory for a long time, which could interfere with the activation and selection of the target.

The PLI group performed similarly to their peers with TD on the visual inhibition task, suggesting that they do not have deficits in inhibiting visuospatial distractors. This finding is consistent with previous research reporting no between-group differences on non-linguistic resistance distractor tasks in school-age children with PLI (Noterdaeme et al., 2001) and on a visuospatial sustained selective attention task in preschoolers with PLI (Spaulding, Plante, \& Vance, 2008). However, the results differ from a recent study demonstrating that school-age children with PLI made more errors than their peers with TD after controlling for age, nonverbal and verbal IQ, and motor response on a nonlinguistic Stroop-like task (Henry et al., 2012).

The mixed reports among these inhibition studies may be due to the different degrees of stimulus-response translation. The tasks used in Noterdaeme et al. (2001) and in our study required children to respond the visual stimuli presented on the screen by pressing either the left or right key. But the Stroop-like task used in Henry et al. (2012) asked children to respond to researcher's hand actions (e.g., a pointed finger versus a fist) 
by making opposite hand actions. The translation between stimulus and response is more natural in the latter than the former: imitating hand actions is a well-learned response to seeing hand actions, whereas pressing a particular key on a keyboard in response to a visual stimulus presented on the screen is arbitrary. Incongruent stimulus attributes create more interference and larger effects when the stimulus-response translation involves a well-learned response (Sugg \& McDonald, 1994). Thus, perhaps between-group differences were reduced by the key pressing response in Noterdaeme et al. (2001) and in our study. It would be interesting to investigate different degrees of stimulus-response translation in children with PLI on non-linguistic inhibition tasks to determine whether there is evidence for nonverbal inhibition deficits under certain experimental conditions.

We did not find between-group differences for RTs (correct responses only) on either type of inhibition task. These results suggest that when children with PLI were be able to inhibit the distractors, they did so as quickly as their peers with TD. Our RT results are consistent with a meta-analyses of sustained attention in children with PLI, which found little support for the claim that children with PLI perform more slowly than their peers with TD on tasks of sustained attention (Ebert \& Kohnert, 2011). One could argue that the PLI group had fewer RTs than the TD group because of accuracy differences, but the accuracy for both groups on both tasks was quite high (88\% and $75 \%$ for the TD and PLI groups, respectively). One possible reason we did not observe between-group difference in RTs may have been that RT results are more sensitive indicators for detecting a conflict effect than accuracy in adults (because of their high accuracy), but young children are less predictable and less consistent in the speed of their RTs than adults. Moreover, children with PLI tend to have extensive variability in their 
speed of response (Windsor, Milbrath, Carney, \& Rakowski, 2001). Thus, variability may contribute to the lack of significant between-group differences for RTs.

We must also acknowledge that the internal consistency estimates of reliability for the inhibition tasks were lower than for other tasks, most likely because of the correlations between congruent and incongruent trials for both accuracy and RTs. The significant correlation implies that these two types of trials were measuring a similar construct. Posner and Rothbart (2001) proposed that attention plays a critical role in conflict resolution and the development of executive function during preschool period. Thus, attention may be the source of common variance underlying these two types of stimuli.

\section{Updating}

Our study was the first to examine updating in preschoolers with PLI. Based on previous research we hypothesized that children with PLI have a general updating deficits; therefore, they would perform less accurately than their peers with TD on both the linguistic and visual updating tasks. Our results supported this hypothesis by showing that the PLI group made significantly more errors than the TD group on both of the updating tasks. These results suggest that their updating deficits are not restricted to the linguistic domain, but also extends to the visuospatial domain of working memory.

To complete our linguistic one-back task children were required to first retrieve the phonological and semantic representations of the linguistic item presented on the screen (e.g., car) and to hold those representations in their working memory for two seconds. Since each stimulus could be labeled linguistically, it could also be rehearsed verbally in working memory. When the next linguistic item was presented (e.g., apple), 
children had to retrieve the representations for the new item and compare the new representations with the previous one to determine whether they were the same or different. It is critical for children to replace the representations of the old item (car) with the new one (apple) in working memory so that they can compare it to the next item (e.g., horse). Thus, the task required continuous on-line updating of activated semantic and phonological representations in working memory, which may have been challenging for the PLI group because of their difficulties in retrieving, maintaining and comparing phonological and semantic representations within the linguistic domain. Updating difficulty has been documented by other studies of preschool-age children with PLI, showing that they performed poorly on verbal storage (Vugus et al., 2014; Petruccelli et al., 2012) and verbal central executive function tasks (Vugus et al., 2014). There is also a large body of evidence indicating that school-age children with PLI exhibit weakness in storing and processing linguistic information simultaneously on verbal working memory tasks, such as listening span recall (Archibald \& Gathercole, 2006; Marton et al., 2007; Henry et al., 2012).

Our results also suggest that preschoolers with PLI exhibit deficits in continuously updating visual information. The visual one-back task required children to update the location of a butterfly on the screen. These locations were hard to label linguistically or to rehearse, which placed a greater demand on visuospatial working memory than linguistic working memory. Their poor performance on this task suggests that preschoolers with PLI have a weakness in updating visuospatial information in working memory. However, it must be acknowledged that this task required children to reply verbally; thus, it was not without some degree of linguistic demand. 
There has been a debate regarding whether children with PLI demonstrate working memory deficits in the visuospatial domain along with their deficits in the verbal domain. Contradictory evidence has been found among empirical results regarding the visuospatial working memory in both school- and preschool-age children with PLI. Some studies report that children with PLI performed similarly to their peers with TD on visuospatial short-term memory and working memory tasks (Archibald \& Gathercole, 2006; Petruccelli et al., 2012), while others indicated that children with PLI scored lower and develop more slowly in this domain than their age-matched peers with TD (Marton et al., 2012; Henry et al., 2012; Vugs et al., 2014). Our findings add credence to these results, showing that preschoolers with PLI have a more general updating deficit.

\section{Shifting}

Ours is the first study to examine mental set shifting in preschool-age children with PLI. Most evidence to date suggests that school-age children with PLI do not have deficits in shifting between mental sets; therefore, we hypothesized that preschoolers with PLI may not have general shifting deficits. Contrary to this hypothesis, our results showed that the PLI group exhibited deficits on both linguistic and visual shifting tasks, providing evidence of a general shifting deficit.

Preschoolers with PLI performed significantly less accurately on the linguistic shifting task than their peers with TD when they were asked to shift from sorting stimuli by one dimension to the other. Successful shifting required children not only update and maintain the current sorting rule in working memory, but also to inhibit attention to the prior dimension and the prior activated mental set (stimulus-response relation) to shift and develop a new mental set (Diamond, 2006; Garon et al., 2008). Since we reduced the 
working memory load in our study by reminding children of the current sorting dimension of the stimulus each trial, their weakness in shifting could come from two sources. The first could be poor resistance to proactive linguistic interference (Marton et al., 2012; Marton et al., 2007). Although we decreased the inhibition load for this task by separating the two dimensions into foreground and background (e.g., shape of glasses on a red background) instead of integrating them together (i.e., a red rabbit), children still needed to inhibit the focus of attention from the one dimension to the other dimension, as well as inhibit the previously activated linguistic mental set (stimulus-response relation). The inability to inhibit over linguistic proactive interference would prevent children with PLI from shifting and developing a new mental set. The second weakness in shifting could be due to language impairment. Because the stimuli could be labeled, the TD group may have had the advantage of labeling the relevant dimension used for sorting to help them inhibit the irrelevant dimension and maintain the current rule. This could help them regulate the process of shifting, but because of their linguistic deficit children in the PLI group may have not been able to use the linguistic information to their advantage. This weakness in shifting between mental sets in children with PLI has also been found in a study when children were asked to constantly change rules for sorting cards (Marton, 2008). Marton reported that school-age children with PLI made more errors, showed more perseverations, and had more difficulty developing a clear sorting principle than their peers with TD.

At first glance the lack of a significant between-group difference on the visual shifting task could suggest that children with PLI do not have a general shifting deficit; however, when we take a closer look at the results, there were two critical indicators to 
suggest a general shifting deficit in the PLI group. The first indicator was that almost half of the children with PLI could not exceed the $50 \%$ chance level for accuracy in the preshifting block, especially when they were asked to sort stimuli by the dimension of "pattern." This inability to sort the stimuli by pattern may be because children with PLI stayed with the more perceptually salient feature, "shape," rather than the "pattern" of the stimuli and they did not have sufficient inhibitory control, or shifting ability in general, to flexibly direct attention to another dimension. This inability to shift to a second dimension or attribute, (known as attentional inertia; Kirkham et al., 2003), has been widely observed in three-year-olds' performance on a DCCS task, but is gradually overcome by four- and five-year-olds with TD. In this study, children with PLI may still be exhibiting attentional inertia. Alternatively, since children with PLI may have had a difficult time mapping linguistic labels to referents (Gray, 2004) and may have had a poor conceptual understanding of the relatively abstract meaning of "pattern.” Perhaps they were unable to map the word "pattern" to the pattern dimension of the stimuli, which hindered them from developing a strong stimulus-response association between patterns and actions. Thus, their insufficiently developed inhibitory control, poor shifting in general, and poor language contributed to their difficulty sorting the stimuli by pattern in the pre-shifting block.

The second critical indicator of a general shifting deficit in preschoolers with PLI was that the mean percentage correct in the post-shifting block $(28.6 \%)$ was below the $50 \%$ chance level, whereas the mean for the TD group was higher than chance $(57.4 \%)$. These results suggest that children with PLI stuck to the initial sorting rule and rarely shifted to the other sorting rule; whereas, children with TD could form a new, but weak 
stimuli-response association for the post-shifting block. Additionally, only one out of twelve children with PLI actually shifted (criterion for success was five or more out of six responses correct post-shift) as opposed to 13 out of 28 children with TD. This shows that most children with PLI could not shift to the new rule and thus demonstrated a general shifting deficit.

Taken together, our shifting results suggest that preschoolers with PLI exhibited a general shifting deficit and that this deficit may partially result from the poor resistance of proactive interference. Although our results differ from most of the studies examining shifting in school-age children with PLI, this inconsistency may be due to different dependent variables. The dependent variable of those studies was the RT difference between shift and non-shift trials; whereas, the dependent variable of our study was the accuracy of post-shift trials. We did not use RT as our dependent variable because there were too few correct responses in post-shift trials to provide enough valid RTs. A future question is to determine why, if preschoolers with PLI have general shifting difficulties, they don't exhibit shifting deficits during their school-age years. Since shifting depends heavily on inhibition and working memory, it could be that with improved inhibition, working memory, and language, children with PLI are better able to shift between mental sets as they mature.

Overall, our results showed that preschoolers with PLI exhibit general executive function deficits when compared to their peers of the same age. This could be because their different executive function are on a slower developmental trajectory and at some point they will catch up to their peers who are on a faster developmental course. Based on the evidence, inhibition may develop earlier than updating and mental set shifting 
(Diamond, 2006). Children with PLI may catch up with their peers on their inhibition, but are still struggled with the late-developed updating and shifting. But if we take the previous evidence of school-age children with PLI into account, they may have persistent executive function deficits. Further longitudinal research is needed to investigate their developmental trajectory.

\section{Limitations}

There were two limitations of our experimental design. One limitation was that the response modes were different for the linguistic and visual inhibition tasks and in some cases this prevented a direct comparison of linguistic and visual results. In the future it may be possible to use the same response mode for both types of tasks. A second limitation is that we used a cross-sectional design which does not permit us to determine whether the PLI group may be exhibiting a developmental delay in some areas of executive function relative to their peers with $\mathrm{TD}$, or whether they have persistent deficits. A longitudinal research design documenting executive function development in children with PLI from three to six years of age would give us a more detailed developmental profile.

\section{Conclusions \& Implications}

In summary, the current study is one of the first to comprehensively assess three core executive functions in young children with PLI using both linguistic and visuospatial tasks. Preschoolers with PLI showed difficulties on a broad range of linguistic and visual executive function tasks, even though participants did not have diagnosed ADHD and scores on an attention scale measure were covaried in our analyses. Specific areas of executive function deficits included linguistic inhibition, linguistic and visual updating, 
and linguistic and visual shifting. Overall, findings add to evidence showing that executive functioning deficits in preschoolers with PLI is not limited to the language domain, but is more general in nature.

The present findings have several potential clinical implications for intervention with preschoolers with PLI. First research is increasingly showing the children with PLI may have deficits in several developmental areas; thus, early intervention should not only focus on language development, but also on developing strategies that will help young children process and store linguistic and visuospatial information. Second, recent research suggests that executive functions and working memory may be enhanced via computerized games, interventions (Wener \& Archibald, 2011) and curriculum (Diamond, Barnett, Thomas, \& Munro, 2007). Moreover, recent findings suggest that working memory-based interventions may improve language, reading, and working memory skills in school-age children with language impairment (Farquharson \& Franzluebbers, 2014). Clinicians must consider assessing and targeting executive function as part of their strategy for improving language and learning in children as young as four years of age. 


\section{REFERENCES}

Alarcon-Rubio, D., Sanchez-Medina, J. A., \& Prieto-Garcia, J. R. (2013). Executive function and verbal self-regulation in childhood: developmental linkages between partially internalized private speech and cognitive flexibility. Early Childhood Research Quarterly, 29, 95-105.

Anderson, P. (2002). Assessment and development of executive function (EF) during childhood. Child Neuropsychology, 8(2), 71-82.

Anderson, V., Jacobs, R., \& Anderson, P. J. (2008). Executive functions and the frontal lobes. New York: Psychology Press.

Archibald, L. M., \& Gathercole, S. E. (2006). Short-term and working memory in specific language impairment. International Journal of Language and Communication Disorders, 41(6), 675-693.

Baddeley, A. D. (1986). Working memory. New York: Oxford University Press.

Baddeley, A. D. (2003). Working memory and language: an overview. Journal of Communication disorders, 36, 189-208.

Baddeley, A. D. (2012). Working memory: theories, models, and controversies. Annual Review of Psychology, 63, 1-29.

Beck Mutch, P. J. (2001). Comparison of performance on test of executive functions between children with and without specific language impairment (Doctoral dissertation). Retrieved from Proguest.

Best, J. R., \& Miller, P. H. (2010). A developmental perspective on executive function, Child Development, 81(6), 1641-1660.

Bishop, D., \& Norbury, C. (2005). Executive functions in children with communication impairments, in relation to autistic symptomatology. Autism, 9, 29-43.

Bjorklund, D. F., \& Hamishfeger, K. K. (1990). The resources construct in cognitive development: diverse sources of evidence and a theory of inefficient inhibition. Developmental Review, 10(1), 48-71.

Blair, C., \& Razza, R. P. (2007). Relating effortful control, executive function, and false belief understanding to emerging math and literacy ability in kindergarten. Child Development, 78(2), 647-663.

Brock, L. L., Rimm-Kaufman, S. E., \& Nathanson, L. (2009). The contributions of 'hot' and 'cool' executive function to children's academic achievement and learningrelated behaviors, and engagement in kindergarten. Early Childhood Research Quarterly, 24(3), 337-349. 
Carlson, S., Martinkauppi, S., Rama, P., Salli, E., Korvenoja, A., \& Aronen, H. J. (1998). Distribution of cortical activation during visuospatial n-back tasks as revealed by functional magnetic resonance imaging. Cerebral Cortex, 8(8), 743-752.

Carlson, S., Mandell, D. J., \& Williams, L. (2004). Executive function and theory of mind: Stability and prediction from ages 2 to 3. Developmental Psychology, 40, 1105-1122.

Carlson, S. M., Moses, L., \& Breton, C. (2002). How specific is the relation between executive function and theory of mind? Contributions of inhibitory control and working memory. Infant and Child Development, 11, 73-92.

Carlson, S. M. (2005). Developmentally sensitive measures of executive function in preschool children. Developmental Neuropsychology, 28, 595-616.

Cohen, J. (1988). Statistical power analysis for behavioral sciences, second edition. NJ: Lawrence Erlbaum Associates, Inc.

Collette, F., \& der Linden, M. V. (2002). Brain imaging of the central executive component of working memory. Neuroscience \& Biobehavioral Reviews, 26(2), 105-125.

Cummings, A., \& Ceponiene, R. (2010). Verbal and nonverbal semantic processing in children with developmental language impairment. Neuropsychologia, 48(1), 77-85.

Davidson, M. C., Amso, D., Anderson, L. C., \& Diamond, A. (2006). Development of cognitive control and executive functions from 4 to 13 years: evidence from manipulations of memory, inhibition, and task switching. Neuropsychologia, 44(11), 2037-2078.

Diamond, A. (2002). Normal development of prefrontal cortex from birth to young adulthood: Cognitive functions, anatomy, and biochemistry. In D. Stuss \& R. Knight (Eds.), Principles of frontal lobe function (pp. 466-503). New York: Oxford University Press

Diamond, A. (2006). The early development of executive functions. In E. Bialystock \& F. I. M. Craik (Eds.), The early development of executive functions. Lifespan cognition: Mechanisms of change (pp. 70-95). Oxford, England: Oxford University Press.

Diamond, A., Barnett, W. S., Thomas, J., \& Munro, S. (2007). Preschool program improves cognitive control. Science, 318, 1387-1388.

Diamond, A., Carlson, S. M., \& Beck, D. M. (2005). Preschool children's performance in task switching on the dimensional change card sort task: separating the dimensions aids the ability to switch. Developmental Neuropsychology, 28(2), 689-729. 
Diamond, A., \& Kirkham, N. (2005). Not quite as grown-up as we like to think parallels between cognition in childhood and adulthood. Psychological Science, 16(4), 291297.

Dibbets, P., Bakker, K., \& Jolles, J. (2006). Functional MRI of task switching in children with specific language impairment (SLI). Neurocase, 12, 71-79.

Dowell, K., \& Bavin, E. L. (2008). Children with specific language impairment: an investigation of their narratives and memory. International Journal of Language and Communication Disorders, 43(2), 201-18.

Dunn, L. M., \& Dunn, L. M. (1997). Peabody Picture Vocabulary Test (3rd ed.). Circle Pines, MN: American Guidance Service.

Ebert, K.D., \& Kohnert, K. (2011). Sustained attention in children with primary language impairment: A meta-analysis. Journal of Speech, Language, and Hearing Research, $54,1372-1384$.

Eckner, J. T., Kutcher, J. S., Richardson, J. K., (2010). Pilot evaluation of a novel clinical test of reaction time in national collegiate athletic association division football player. Journal of Athletic Training, 45(4), 327-332.

EllisWeismer, S., Evans, J., \& Hesketh, L. (1999). An examination of verbal working memory capacity in children with specific language impairment. Journal of Speech, Language, and Hearing Research, 42, 1249-1260.

Ellis Weismer, S., Plante, E., Jones, M., \& Tomblin, J. B. (2005). A functional magnetic resonance imaging investigation of verbal working memory in adolescents with specific language impairment. Journal of Speech, Language, and Hearing Research, 48, 405-425.

Espy, K. A. (2004). Introduction: Executive control in preschool children. Developmental Neuropsychology, 26, 379-384.

Espy, K. A., Kaufmann, P., Glisky, M., \& McDiarmid, M. (2001). New procedures to assess executive functions in preschool children. Clinical Neuropsychologist, 15 , $46-58$.

Farquharson, K., \& Franzluebbers, C. E. (2014). Comparing the effects of working memory-based interventions for children with language impairment. EBP Briefs, 9(1), 1-7. Bloomington, MN: Pearson.

Fenson, L., Dale, P., Reznick, S. J., Bates, E., Thal. D. J., \& Pethick, S. J., (1994). Variability in Early Communication Development. Monographs of the society for research in child development, 59(5). 
Finneran, D. A., Francis, A. L., \& Beonard, L. B. (2009). Sustained attention in children with specific language impairment. Journal of Speech, Language, and Hearing Research, 52, 915-929.

Foster, K., \& Forster, J. C. (2003). DMDX: A windows display program with millisecond accuracy. Behavior Research Methods, Instruments, \& Computers, 35(1), 116-124.

Friedman, N. P., \& Miyake, A. (2004). The relations among inhibition and interference control functions: a latent-variable analysis. Journal of Experimental Psychology: General, 133(1), 101-135.

Friedman, N. P., Miyake, A., Corley, R.P., Young, S.E., DeFries, J.C., \& Hewitt, J.K. (2006). Not all executive functions are related to intelligence. Psychological Science, $17,172-179$.

Friedman, N. P., Miyake, A., Robinson, J. A. L., \& Hewitt, J. K. (2011). Developmental trajectories in toddlers' self-restraint predict individual differences in executive functions 14 years later: a behavioral genetic analysis. Developmental Psychology, 47(5), 1410-1430.

Frye, D., Zelazo, P., \& Palfai, T. (1995). Theory of mind and rule-based reasoning. Cognitive Development, 10, 483-527.

Fuhs, M. W., \& Day, J. D. (2011). Verbal ability and executive functioning development in preschoolers at head start. Developmental Psychology, 47, 404-416.

Garon, N., Bryson. S. E., \& Smith, I. M. (2008). Executive function in preschoolers: A review using an integrative framework. Psychological Bulletin, 134, 31-60.

Gathercole, S. (1998). The development of memory. Journal of Child Psychology and Psychiatry, 39, 3-27.

Gioia, G. A., Espy, K. A., \& Isquith, P. K., (2003). Behavior rating inventory of executive function, preschool version (BRIEF-P). Odessa, FL: Psychological Assessment Resources.

Gray, S. (2004). Word learning by preschoolers with specific language impairment: predictors and poor learnings. Journal of Speech, Language, and Hearing Research, 47, 1117-1132.

Greenslade, K. J., Plante, E., \& Vance, R. (2009). The diagnostic accuracy and construct validity of the structured photographic expressive language test-preschool: second edition. Language, Speech, and Hearing Services in Schools, 40, 150-160.

Guy, J., Rogers, M., \& Cornish, K. (2012). Developmental changes in visual and auditory inhibition in early childhood. Infant and Child Development, 21(5), 521-536. 
Happaney, K., \& Zelazo, P. D. (2003). Inhibition as a problem in the psychology of behavior. Developmental Science, 6(5), 468-470.

Henry, L. A., Messer, D. J., \& Nash, G. (2012). Executive functioning in children with specific language impairment. Journal of Child Psychology and Psychiatry, 53(1), $37-45$.

Hoffman, L. M., \& Gillam, R. B. (2004). Verbal and spatial information processing constraints in children with specific language impairment. Journal of Speech, Language, and Hearing Research, 47, 114-125.

Homer, B. D., Petroff, N., \& Hayward, E. O. (2008). Linguistic mediation of children's performance in a new symbolic understanding task. Journal of Cognition and Development, 14(3), 455-466.

Hughes, C. (1998). Executive function in preschoolers: Links with theory of mind and verbal ability. British Journal of Developmental Psychology, 16(2), 233-253.

Huizinga, M., Dolan, C. V., \& der Molen, M. W. (2006). Age-related change in executive function: developmental trends and a latent variable analysis. Neuropsychologia, 44, 2017-2036.

Im-Bolter, N., Johnson, J., \& Pascual-Leone, J. (2006). Processing limitations in children with specific language impairment: The role of executive function. Child Development, 77, 1822-1841.

Isquith, P. K., Gioia, G., \& Espy, K. A. (2004). Executive function in preschool children: Examination through everyday behavior. Developmental Neuropsychology, 26(1), 403-422.

Johnstone, S. J., Dimoska, A., Smith, J. L., Barry, R. J., Pleffer, C. B., Chiswich, D., \& Clarke, A. R. (2007). The development of stop-signal and Go/Nogo response inhibition in children aged 7-12 years: performance and event-related potential indices. International Journal of Psychophysiology, 63(1), 25-38.

Kane, M. J., \& Engel, R. W. (2002). The role of prefrontal cortex in working memory capacity, executive attention, and general fluid intelligence: an individualdifferences perspective. Psychonomic Bulletin \& Review, 9, 637-671.

Kaufman, A. S., \& Kaufman, N. L. (2004). Kaufman Brief Intelligence Test, Second Edition. Bloomington, MN: Pearson, Inc.Kirkham, N. Z., Cruess, L., \& Diamond, A. (2003). Helping children apply their knowledge to their behavior on a dimensionswitching task. Developmental Science, 6(5), 449-476.

Kline, P (2000). Handbook of psychological testing. New York: Routledge. 
Kloo, D., \& Perner, J. (2005). Disentangling dimensions in the dimensional change chard-sorting task. Developmental Science, 8(1), 44-56.

Kirkham, N. Z., Cruess, L., \& Diamond, A. (2003). Helping children apply their knowledge to their behavior on a dimension-switching task. Developmental Science, 6(5), 449-476.

Kuntz, B. P. (2012). Selective attention in children with specific language impairment: auditory and visual Stroop effects (Doctoral dissertation). Request from Proguest.

Lee, K., Bull, R., \& Ho, R. M. H. (2013). Developmental changes in executive functioning. Child Development, 84(6), 1933-1953.

Lehto, J. E., Juujarvi, P., Kooistra, L., \& Pulkkinen, L. (2003). Dimensions of executive functioning: evidence from children. British Journal of Developmental Psychology, $21,59-80$.

Lenord, L. B (1998). Children with specific language impairment. Cambridge, MA: MIT Press.

Lyon, G. R., \& Krasnegor, N. A. (1996). Attention, memory, and executive function. MD: Paul H Brookes Publishing.

Marton, K. (2008). Visuo-spatial processing and executive functions in children with specific language impairment. International Journal of Language \& Communication Disorders, 43(2), 181-200.

Marton, K., Kelmenson, L., \& Pinkhasova, M. (2007). Inhibition control and working memory capacity in children with SLI. Psychologia, 50, 110-121.

Marton, K., Campanelli, L., Eichorn, N., Scheuer, J., \& Yoon, J. (2014). Information processing and proactive interference in children with and without specific language impairment. Journal of Speech, Language, and Hearing Research, 57(1), 106-119.

Marton, K., Campanelli, L., Scheuer, J., Yoon, J., \& Eichorn, N. (2012). Executive function profiles in children with and without specific language impairment. Rivista di psicolinguistica applicate, 12(3), 57-73.

Mazuka, R., Jincho, N., \& Oishi, H. (2009). Development of executive function and language processing. Language and Linguistic Compass, 3(1), 59-89.

McClelland, M. M., Cameron, C. E., Connor, C. M., Farris, C. L., Jewkes, A. M., \& Morrison, F. J. (2007). Links between behavioral regulation and preschoolers' literacy, vocabulary, and math skills. Developmental Psychology, 43(4), 947-959. 
Miller, R. M., Giesbrecht, G. F., Müller, U., Mclnerney, R. J., \& Kerns, K. A. (2012). A latent variable approach to determining the structure of executive function in preschool children. Journal of Cognition and Development, 13(3), 395-423.

Miller, C., Kail, R., Leonard, L. B., \& Tomblin, J. B. (2001). Speed of processing in children with specific language impairment. Journal of Speech, Language, and Hearing Research, 44, 416-433.

Miyake, A., Friedman, N. P., Emerson, M. J., Witzki, A. H., Howenter, A., \& Wager, T. D. (2000). The unity and diversity of executive functions and their contributions to complex "frontal lobe" tasks: a latent variable analysis. Cognitive Psychology. 41, 49-100.

Morton, J. B., \& Munakata, Y. (2002). Active versus latent representations: a neural network model of perseveration, dissociation, and decalage. Developmental psychobiology, 40(3), 255-265.

Müller, U., Zelazo, P. D., Lurye, L. E., \& Liebermann, D. P. (2008). The effect of labeling on preschool children's performance in the dimensional change card sort task. Cognitive Development, 23, 395-408.

Munakata, Y. (2001). Graded representations in behavioral dissociations. Trends in Cognitive Sciences, 5, 309-315.

Munakata, Y., Morton, B., \& Yerys, B. (2003). Children's perseveration: Attentional inertia and alternative accounts. Developmental Science, 6, 471-473.

Norman, D. A., \& Shallice, T. (1986). Attention to action: willed and automatic control of behavior. In G. E. Schwartz \& D. Shapiro (Eds.), Consciousness and selfregulation, 4, New York: Plenum Press.

Noterdaeme, M., Amorosa, H., Mildenberger, K., Sitter, S., \& Minow, F. (2001). Evaluation of attention problems in children with autism and children with a specific language disorder. European Child \& Adolescent Psychiatry, 10, 58-66.

Oram, J. E. (2003). Seeking cognitive markers of specific language impairment and attention deficit/hyperactivity disorder (Doctoral dissertation). Retrieved from Proquest.

Petrucelli, N., Bavin, E. L., \& Bretherton, L (2012). Children with specific language impairment and resolved late talkers: working memory profiles at 5 years. Journal of Speech, Language and Hearing Research, 55(6), 1690-1703.

Posner, M. I., \& Rothbart, M. K. (2007). Research on attention networks as a model for the integration of psychological science. Annual Review of Psychology, 58, 1-23. 
Rueda, R. M., Posner, M, I., Rothbart, M. K., \& Davis-Stober, C. P. (2004). Development of the time course for processing conflict: an event-related potentials study with 4 year olds and adults. BMC Neuroscience, 5, 39.

Rypma, B., \& D’Esposito, M. (2000). Isolating the neural mechanisms of age-related changes in human working memory. Nature Neuroscience, 3(5), 509-515.

Schul, R., Stiles, J., Wulfeck, B., \& Townsend, J. (2004). How 'generalized' is the 'slowed processing' in SLI? The case of visuospatial attentional orienting. Neuropsychologia, 42(5), 661-671.

Seiger-Gardner, L., \& Schwartz, R. G. (2008). Lexical access in children with and without specific language impairment: a cross-modal picture-word interference study. International Journal of Language \& Communication Disorders, 43(5), 528551.

Shing, Y. L., Lindenberger, U., Diamond, A., Li, S. C., \& Davidson, M. C. (2010). Memory maintenance and inhibitory control differentiate from early childhood to adolescence. Developmental Neuropsychology, 35(6), 679-697.

Simpson, A., Riggs, K. J., Beck, S. R., Gorniak, S. L., Wu, Y., Abbott, D., \& Diamond, A. (2012). Refining the understanding of inhibitory processes: how response prepotency is created and overcome. Developmental Science, 15(1), 62-73.

Spaulding, T. J. (2010). Investigating mechanisms of suppression in preschool children with specific language impairment. Journal of Speech, Language, and Hearing Research, 53, 725-738.

Spaulding, T. J., Plante, E., \& Vance, R. (2008). Sustained selective attention skills of preschool children with specific language impairment: Evidence for separate attentional capacities. Journal of Speech, Language, and Hearing Research, 51, $16-34$.

Sugg, M. J., \& McDonald, J. E. (1994). Time course of inhibition in color-response and word-response versions of the Stroop task. Journal of Experimental Psychology: Human Perception and Performance, 20, 647-675.

Swanson, J. Schuck, S., Mann, M., Carlson, C., Hartman, K., Sergeant, J., Clevenger, W., Wasdell, M., \& McCleary, R. (2005). Categorical and dimensional definitions and evaluations of symptoms of ADHD: The SNAP and the SWAN Ratings Scales. Available at: http://www.adhd.net/SNAP_SWAN.pdf. Accessed Dec 07, 2013.

Tsujimoto, S. (2008). The prefrontal cortex: functional neural development during early childhood. The Neuroscientist, 14(4), 345-358. 
Tsujimoto, S., Kuwajima, M., \& Sawaguchi, T. (2007). Developmental fractionation of working memory and response inhibition during childhood. Experimental Psychology, 54(1), 30-37.

Tsujimoto, S., Yamamoto, T., Kawaguchi, H., Koizumi, H., \& Sawaguchi, T. (2004). Prefrontal cortical activation associated with working memory in adults and preschool children: an event-related optical topography study. Cerebral Cortex, 14, $703-12$.

Tsujimoto, S., Yamamoto, T., Kawaguchi, H., Koizumi, H., \& Sawaguchi, T. (2003). Functional maturation of the prefrontal cortex in preschool children measured by optical topography. Program No. 196.15. 2003 Abstract Viewer/Itinerary Planner. Washington (DC): Society for Neuroscience.

Vaughan, L., \& Giovanello, K. (2010). Executive function in daily life: Age-related influences of executive processes on instrumental activities of daily living. Psychology and Aging, 25(2), 343-355.

Verbruggen, F., Liefooghe, B., \& Vandierendonck, A. (2004). The interaction between stop signal inhibition and distractor interference in the flanker and Stroop task. Acta Psychologica, 116(1), 21-37.

Vugs, B., Cuperus, J., Hendriks, M., \& Verhoeven, L. (2013). Visuospatial working memory in SLI: a meta-analysis. Research in Developmental Disabilities, 34(9), 2586-2597.

Vugs, B., Hendriks, M., Cuperus, J., \& Verhoeven, L. (2013). Working memory performance and executive function behaviors in young children with SLI. Research in Developmental Disabilities, 35, 62-74.

Vygotsky, L. S. (1987). Thinking and speech. In R. W. Rieber, \& A. S. Carton (Eds.), The collected works of L.S. Vygotsky: Vol. 1. Problems of general psychology (pp. 37-285). New York, NY: Plenum (original work published 1934).

Wechsler, D. (2002). Wechsler preschool and primary scale of intelligence TM, Third Edition (WPPSITM-III). TX: Psychological Corporation.

Welsh, J. A., Nix, R. L., Blair, C., Bierman, K. L., \& Nelson, K. E. (2010). The development of cognitive skills and gains in academic school readiness for children from low-income families. Journal Education Psychology, 102(1), 43-53.

Wener, S. E., \& Archibald, L. M. (2011). Domain-specific treatment effects in children with language and/or working memory impairments: a pilot study. Child Language Teaching and Therapy, 27(3), 313-330. 
Willoughby, M. T., Blair, C. B., Wirth, R. J., \& Greenberg, M. (2012). The measurement of executive function at age 5: psychometric properties and relationship to academic achievement. Psychological Assessment, 24(1), 226-239.

Wiebe, S. A., Espy, K. A., \& Charak, D. (2008). Using confirmatory factor analysis to understand executive control in preschool children: I. Latent structure. Developmental Psychology,44, 575-587.

Wiebe, S. A., Sheffield, T., Nelson, J. M., Clark, C. A. C., Chevalier, N., \& Espy, K. A. (2011). The structure of executive function in 3-year-old children. Journal Experimental Child Psychology, 108(3), 436-452.

Wiig, E., Secord,W., \& Semel, E. (1992). Clinical Evaluation of Language Fundamentals-Preschool. San Antonio, TX: The Psychological Corporation.

Windsor, J., \& Kohnert, K. (2009). Processing speed, attention, and perception in child language disorders. In R. T. Schwartz (Eds.), Handbook of Child Language Disorders (p. 445-461). NY: Psychology Press.

Windsor, J., Milbrath, R. L., Carney, E. J., \& Rakowski, S. E. (2001). General slowing in language impairment: Methodological considerations in testing the hypothesis. Journal of Speech, Language, and Hearing Research, 44, 446-461.

Wittke, K., Spaulding, T. J., \& Schechtman, C. J. (2013). Specific language impairment and executive functioning: parent and teacher ratings of behavior. American Journal of Speech-Language Pathology, 22, 161-172.

Zelzao, P. D., Frye, D., \& Rapus, T. (1996). An age-related dissociation between knowing rules and using them. Cognitive Development, 11(1), 37-63.

Zelazo, P. D. (1999). Language, levels of consciousness, and the development of intentional action. In P. D. Zelazo, J. W. Astington, D. R. Olson (Eds.), Developing theories of intention: Social understanding and self-control (pp. 95-117). NJ: Lawrence Erlbaum Associates.

Zelazo, P. D., \& Frye, D. (1998). Cognitive Complexity and Control: II. The Development of Executive Function in Childhood. Current Directions in Psychological Science, 7(4), 121-126.

Zelzao, P, D., Frye, D., \& Rapus, T. (1996). An age-related dissociation between knowing rules and using them. Cognitive Development, 11(1), 37-63.

Zelazo, P. D., Müller, U., Frye, D., \& Marcovitch, S. (2003). The development of executive function in early childhood. Monographs of the Society for Research in Child Development, 68, 1-137. 


\section{APPENDIX A}

ARIZONA STATE UNIVERSITY'S

INSTITUTIONAL REVIEW BOARD APPROVAL 


\begin{tabular}{|c|c|}
\hline & Office of Research Integrity and Assurance \\
\hline To: & $\begin{array}{l}\text { Shelley Gray } \\
\text { COOR }\end{array}$ \\
\hline From: & $\begin{array}{l}\text { for Mark Roosa, Chair mm } \\
\text { Soc Beh IRB }\end{array}$ \\
\hline Date: & $09 / 09 / 2013$ \\
\hline Committee Action: & Amendment to Approved Protocol \\
\hline Approval Date: & 09/09/2013 \\
\hline Review Type: & Expedited F6 F7 \\
\hline IRB Protocol \#: & 1304009148 \\
\hline Study Title: & $\begin{array}{l}\text { Impact of Executive Function on Early Literacy Skills in Pre-Kindergarteners with } \\
\text { and without Primary Language Impairment (PLI) }\end{array}$ \\
\hline Expiration Date: & $05 / 20 / 2014$ \\
\hline \multicolumn{2}{|c|}{$\begin{array}{l}\text { The amendment to the above-referenced protocol has been APPROVED following Expedited Review by the } \\
\text { Institutional Review Board. This approval does not replace any departmental or other approvals that may be } \\
\text { required. It is the Principal Investigator's responsibility to obtain review and continued approval of ongoing } \\
\text { research before the expiration noted above. Please allow sufficient time for reapproval. Research activity of } \\
\text { any sort may not continue beyond the expiration date without committee approval. Failure to receive approval } \\
\text { for continuation before the expiration date will result in the automatic suspension of the approval of this protoco } \\
\text { on the expiration date. Information collected following suspension is unapproved research and cannot be } \\
\text { reported or published as research data. If you do not wish continued approval, please notify the Committee of } \\
\text { the study termination. }\end{array}$} \\
\hline
\end{tabular}

This approval by the Soc Beh IRB does not replace or supersede any departmental or oversight committee review that may be required by institutional policy.

Adverse Reactions: If any untoward incidents or severe reactions should develop as a result of this study, you are required to notify the Soc Beh IRB immediately. If necessary a member of the IRB will be assigned to look into the matter. If the problem is serious, approval may be withdrawn pending IRB review.

Amendments: If you wish to change any aspect of this study, such as the procedures, the consent forms, or the investigators, please communicate your requested changes to the Soc Beh IRB. The new procedure is not to be initiated until the IRB approval has been given.

Please retain a copy of this letter with your approved protocol. 


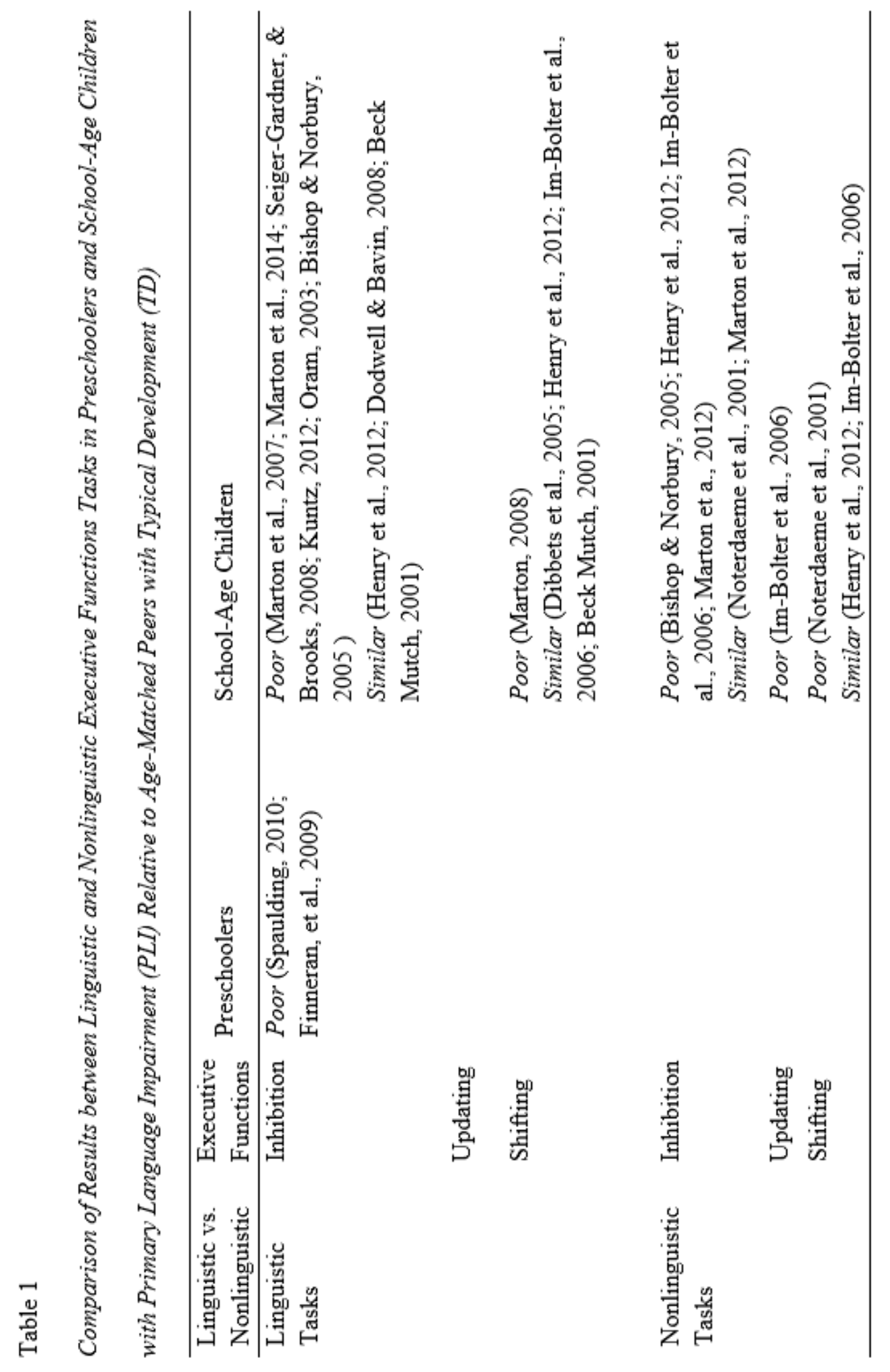


Table 2

Participant Description Information Including Summary of Inclusionary Test Results

\begin{tabular}{|c|c|c|c|c|}
\hline \multirow{2}{*}{ Measure } & \multicolumn{2}{|c|}{$\begin{array}{c}\text { PLI group } \\
(n=22 ; 13 \text { boys, } 9 \text { girls })\end{array}$} & \multicolumn{2}{|c|}{$\begin{array}{c}\text { TD group } \\
(n=30 ; 12 \text { boys, } 18 \text { girls })\end{array}$} \\
\hline & $M$ & $S D$ & $M$ & $S D$ \\
\hline Age in months & 56.77 & 5.23 & 57.30 & 5.67 \\
\hline Mother's Education (in years) & $14.45^{\mathrm{c}}$ & 1.82 & 15.83 & 1.80 \\
\hline Matrices Subtest of K-BIT ${ }^{\mathrm{a}}$ & 102.77 & 9.97 & 108.80 & 10.78 \\
\hline SPELT-P2 ${ }^{\mathrm{a}}$ & $77.09^{c}$ & 8.97 & 106.6 & 7.03 \\
\hline ADHD Scale ${ }^{b}$ & $8.23^{c}$ & 4.60 & 3.14 & 2.67 \\
\hline
\end{tabular}

Note. PLI= primary language impairment; TD = Typical development; K-BIT = Kaufman Brief Intelligence Test Second Edition (Kaufman \& Kaufman,1990); SPELT-P2 = Structured Photographic Expressive Language Test-Preschool-2 (Dawson, Stout, Eyer, Tattersall, Fonkalsrud, \& Croley, 2004); ADHD scale = ADHD Symptoms and Normal Behavior Scale (Swanson, Schuck, Mann, Carlson, Hartman, Sergeant, Clevenger, Wasdell, \& McCleary, 2005). ${ }^{\text {a }}$ Standard scores with a mean of 100 and a standard deviation of 15 .

${ }^{b}$ Raw scores of the ADHD Scale with a maximum raw score of 18 (the higher score denotes the child is likely having ADHD symdrom)

${ }^{\mathrm{c}}$ Indicates significant group difference $(t$ test) after Bonferroni correction to.01, $p<.01$. 


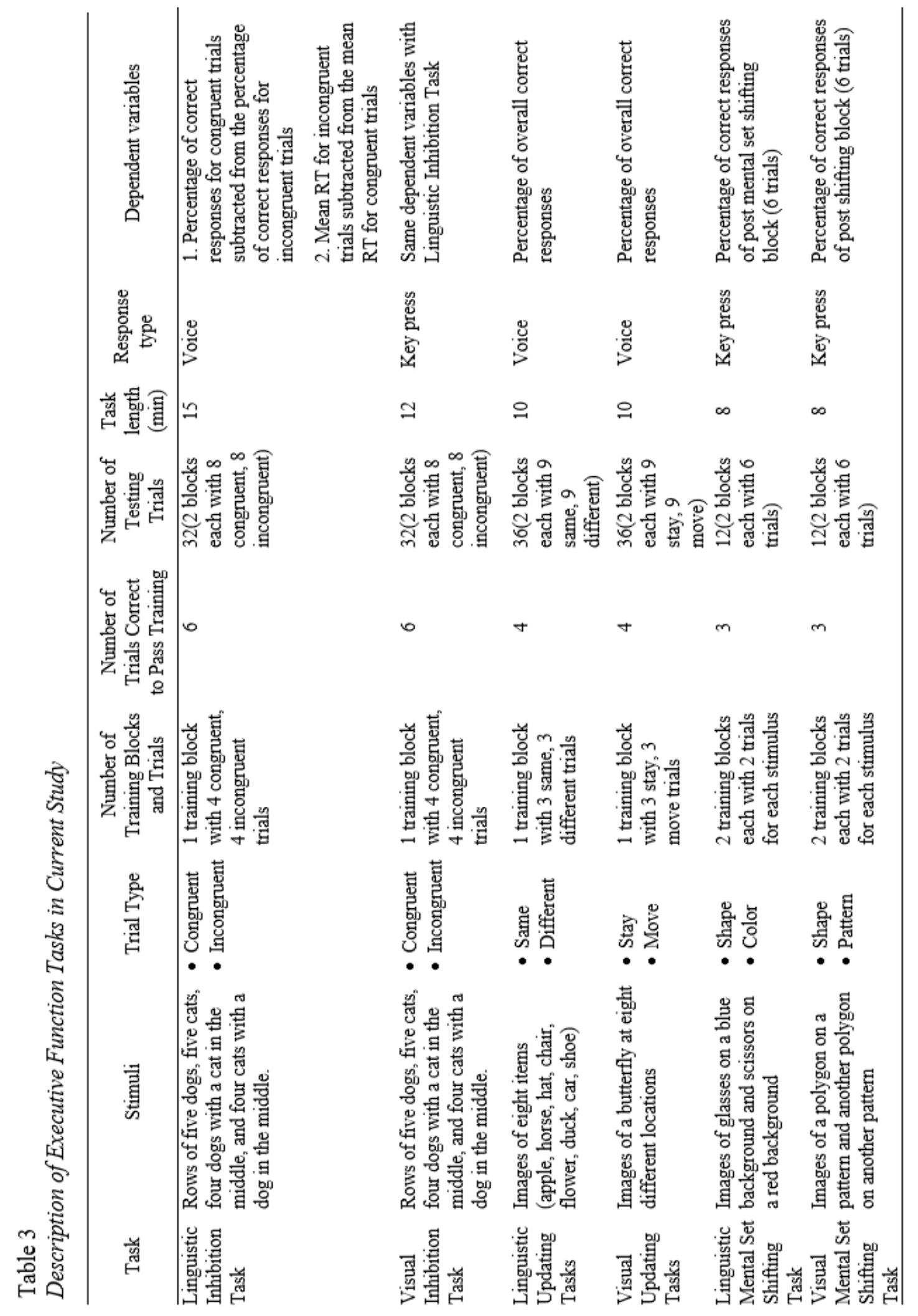


Table 4

Reliability of Executive Function Tasks in Current Study

\begin{tabular}{|c|c|c|c|c|c|c|}
\hline \multirow{2}{*}{ Tasks } & \multicolumn{2}{|r|}{ TD } & \multicolumn{2}{|r|}{ PLI } & \multicolumn{2}{|c|}{ Combined } \\
\hline & $n$ & Reliability & $n$ & Reliability & $n$ & Reliability \\
\hline Motor RT task & 30 & & 22 & & 52 & \\
\hline RT & & .74 & & .77 & & .80 \\
\hline Voice response $\mathrm{RT}$ task & 30 & & 21 & & 51 & \\
\hline RT & & .66 & & .75 & & .74 \\
\hline Linguistic inhibition task & 30 & & 21 & & 51 & \\
\hline Difference accuracy & & .27 & & .31 & & .31 \\
\hline Congruent trials accuracy & & .75 & & .64 & & .73 \\
\hline $\begin{array}{l}\text { Incongruent trials } \\
\text { accuracy }\end{array}$ & & .76 & & .67 & & .77 \\
\hline Difference RT & & .20 & & .37 & & .27 \\
\hline Congruent trials RT & & .79 & & .81 & & .81 \\
\hline Incongruent trials RT & & .26 & & .40 & & .30 \\
\hline Visual inhibition task & 30 & & 22 & & 52 & \\
\hline Difference accuracy & & .68 & & 1.00 & & 1.00 \\
\hline Congruent trials accuracy & & .89 & & .91 & & .90 \\
\hline $\begin{array}{l}\text { Incongruent trials } \\
\text { accuracy }\end{array}$ & & .83 & & .86 & & .86 \\
\hline Difference RT & & .31 & & .95 & & .92 \\
\hline Congruent trials RT & & .93 & & .74 & & .90 \\
\hline Incongruent trials RT & & .94 & & .82 & & .91 \\
\hline Linguistic updating task & 30 & & 15 & & 45 & \\
\hline Overall accuracy & & .81 & & .66 & & .80 \\
\hline Visual updating task & 30 & & 18 & & 48 & \\
\hline Overall accuracy & & .81 & & .81 & & .84 \\
\hline Linguistic shifting task & 30 & & 22 & & 52 & \\
\hline Post-shift block accuracy & & .93 & & .89 & & .92 \\
\hline Linguistic shifting task & 30 & & 22 & & 52 & \\
\hline Post-shift block accuracy & & .93 & & .91 & & .92 \\
\hline
\end{tabular}

Note . $\mathrm{PLI}=$ primary language impairment; $\mathrm{TD}=$ Typical development. 
Table 5

ANOVA Results of Groups on Experimental Tasks

\begin{tabular}{|c|c|c|c|c|c|c|c|c|}
\hline \multirow[b]{3}{*}{ Task/Variable } & \multicolumn{8}{|c|}{ ANOVA } \\
\hline & \multicolumn{2}{|r|}{ TD } & \multicolumn{2}{|r|}{ PLI } & \multirow{2}{*}{$F$} & \multirow{2}{*}{$d f$} & \multirow{2}{*}{$p$} & \multirow{2}{*}{$d$} \\
\hline & $n$ & Mean & $n$ & Mean & & & & \\
\hline \multicolumn{9}{|l|}{ Motor RT Task } \\
\hline $\mathrm{RT}$ & 30 & 1151.28 & 22 & 1385.52 & 12.92 & 1,50 & .001 & 1.01 \\
\hline \multicolumn{9}{|l|}{ Voice RT Task } \\
\hline $\mathrm{RT}$ & 30 & 748.26 & 21 & 904.24 & 10.30 & 1,49 & .001 & .91 \\
\hline \multicolumn{9}{|l|}{ Linguistic Inhibition } \\
\hline \multicolumn{9}{|l|}{ Task } \\
\hline Conflict effect & 30 & 2.92 & 21 & 8.93 & 4.48 & 1,49 & .039 & 60 \\
\hline \multicolumn{9}{|l|}{$\mathrm{ACC}$} \\
\hline Conflict effect RT & 30 & 105.99 & 21 & 126.67 & .22 & 1,49 & .644 & .04 \\
\hline \multicolumn{9}{|l|}{ Visual Inhibition } \\
\hline \multicolumn{9}{|l|}{ Task } \\
\hline $\begin{array}{l}\text { Conflict effect } \\
\text { ACC }\end{array}$ & 30 & 6.15 & 22 & 10.80 & 98 & 1,50 & .328 & .28 \\
\hline Conflict effect RT & 30 & 317.06 & 22 & 484.91 & 2.06 & 1,50 & .158 & .40 \\
\hline \multicolumn{9}{|l|}{ Linguistic Updating } \\
\hline \multicolumn{9}{|l|}{ Tasks } \\
\hline $\mathrm{ACC}$ & 30 & 93.43 & 15 & 80.74 & 15.36 & 1,43 & $<.001$ & 1.24 \\
\hline \multicolumn{9}{|l|}{ Visual Updating Task } \\
\hline $\mathrm{ACC}$ & 30 & 83.15 & 18 & 67.75 & 13.48 & 1,46 & $<.001$ & 1.09 \\
\hline \multicolumn{9}{|l|}{ Linguistic Shifting } \\
\hline Post-shifting ACC & 28 & 74.40 & 19 & 42.98 & 8.89 & 1,45 & .005 & .89 \\
\hline \multicolumn{9}{|l|}{ Visual Shifting Task } \\
\hline Post-shifting ACC & 28 & 59.52 & 12 & 23.61 & 7.89 & 1,38 & .008 & .97 \\
\hline
\end{tabular}

Note. $\mathrm{PLI}=$ primary language impairment; $\mathrm{TD}=$ Typical development; $\mathrm{ACC}=$ accuracy . 


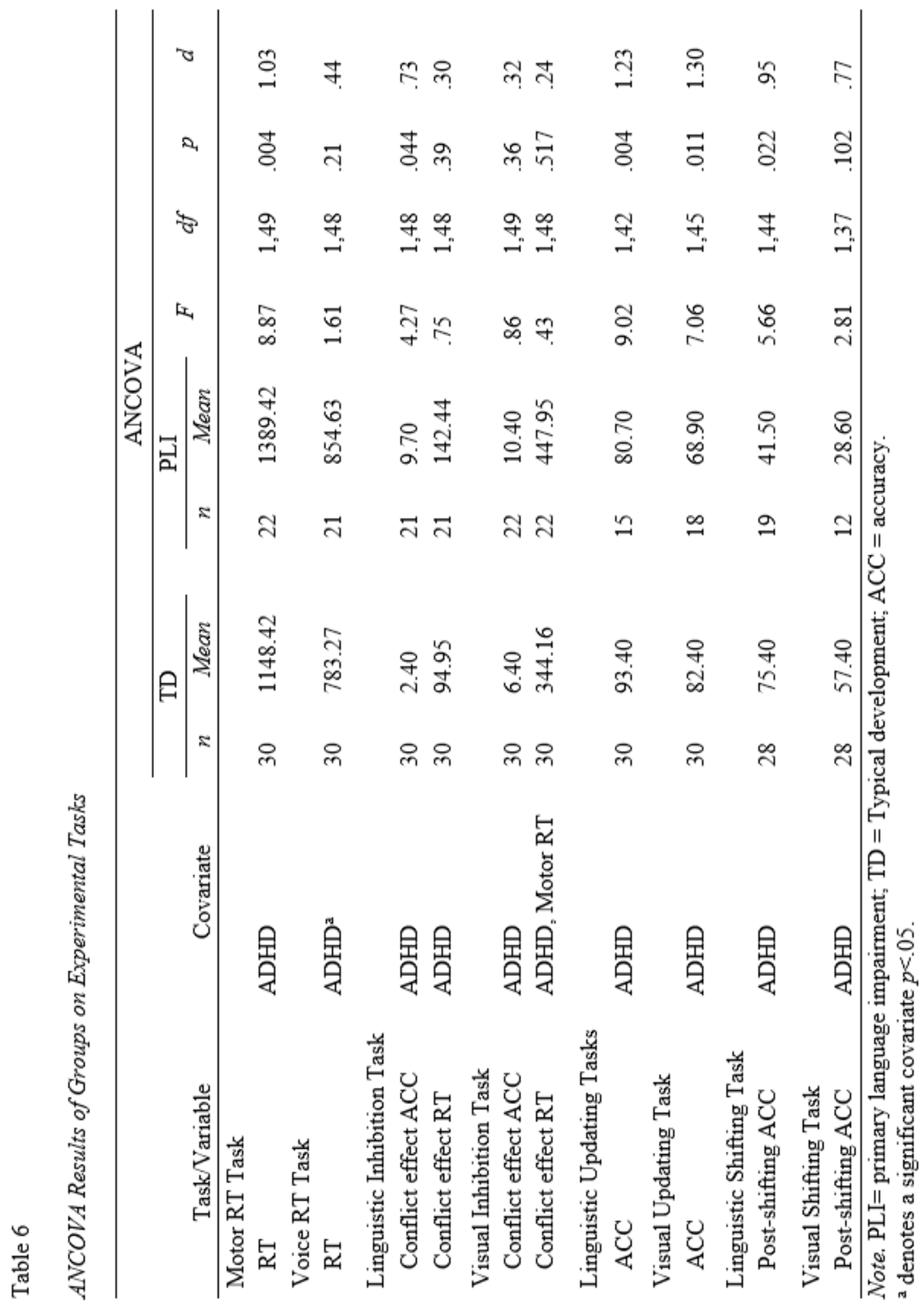




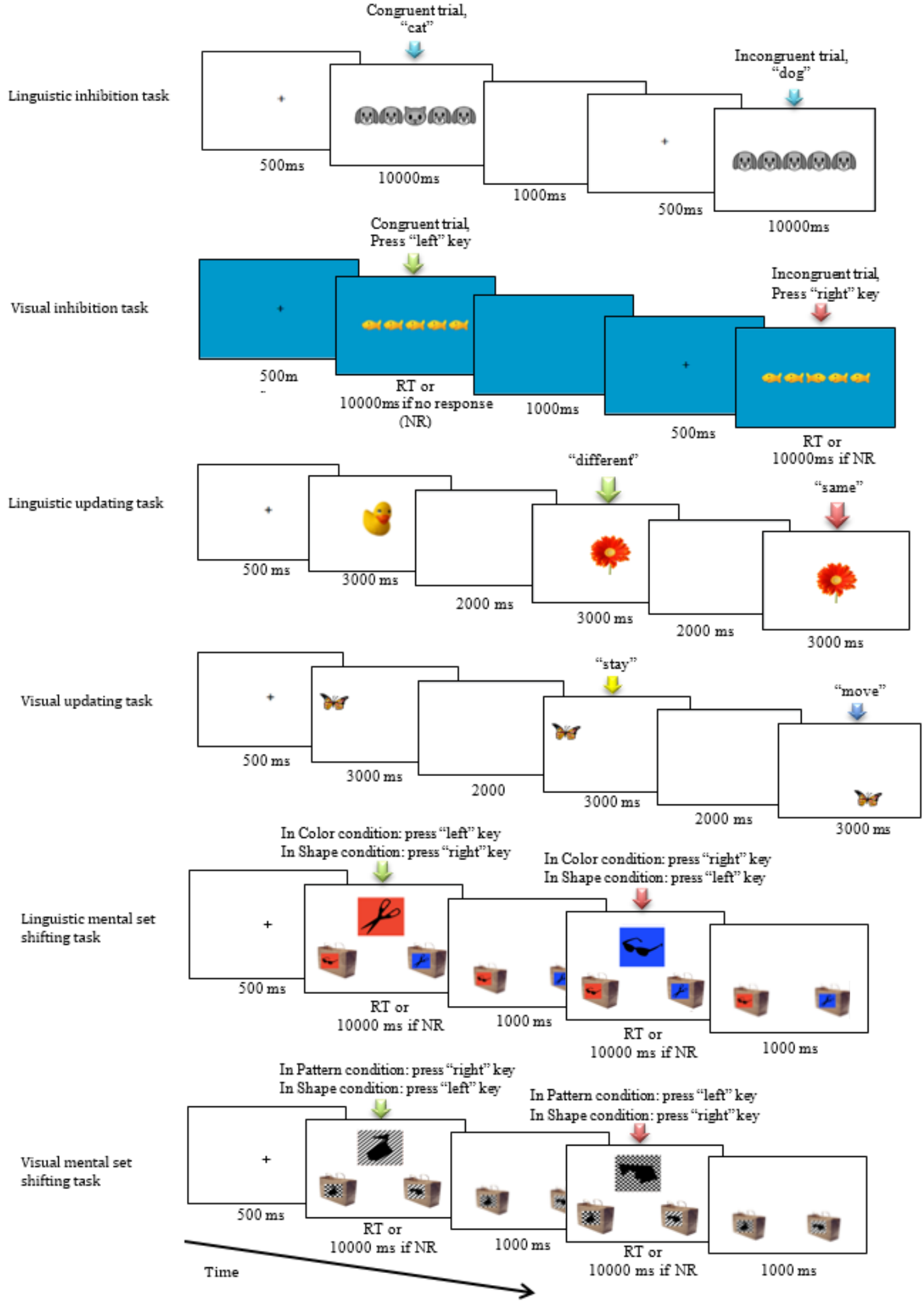

Figure 1. Illustration of executive function tasks used in current study. 